\title{
Knock-down of odr-3 and ife-2 additively extends lifespan and healthspan in C. elegans
}

\author{
Ioan Valentin Matei ${ }^{1,{ }^{*}}$, Vimbai Netsai Charity Samukange ${ }^{1,{ }^{*}}$, Gabriela Bunu$^{1}$, Dmitri Toren ${ }^{1,2}$, \\ Simona Ghenea ${ }^{1, *}$, Robi Tacutu ${ }^{1, *}$ \\ ${ }^{1}$ Systems Biology of Aging Group, Institute of Biochemistry of the Romanian Academy, Bucharest, Romania \\ ${ }^{2}$ The Shraga Segal Department of Microbiology, Immunology and Genetics, Center for Multidisciplinary Research \\ on Aging, Ben-Gurion University of the Negev, Beer-Sheva, Israel \\ *Equal contribution
}

Correspondence to: Simona Ghenea, Robi Tacutu; email: gheneas@biochim.ro; robi.tacutu@gmail.com, https://orcid.org/0000-0001-5853-2470

Keywords: lifespan extension, genetic interventions, synergism, ife-2, odr-3

Received: June 11, $2021 \quad$ Accepted: August 24, $2021 \quad$ Published: September 9, 2021

Copyright: (C) 2021 Matei et al. This is an open access article distributed under the terms of the Creative Commons Attribution License (CC BY 3.0), which permits unrestricted use, distribution, and reproduction in any medium, provided the original author and source are credited.

\begin{abstract}
Genetic manipulations can ameliorate the aging process and extend the lifespan of model organisms. The aim of this research was to identify novel genetic interventions that promote both lifespan and healthspan, by combining the effects of multiple longevity-associated gene inactivations in C. elegans. For this, the individual and combined effects of the odr-3 mutation and of ife-2 and cku-70 knock-downs were studied, both in the wild type and daf-16 mutant backgrounds. We found that besides increasing the lifespan of wild type animals, the knock-down of ife-2 (starting at L4) also extends the lifespan and healthspan of long-lived odr-3 mutants. In the daf-16 background, ife-2 and odr-3 impairment exert opposing effects individually, while the daf-16; odr-3; ife-2 deficient animals show a similar lifespan and healthspan as daf-16, suggesting that the odr-3 and ife-2 effector outcomes converge downstream of DAF-16. By contrast, cku-70 knock-down did not extend the lifespan of single or double odr-3; ife-2 inactivated animals, and was slightly deleterious to healthspan. In conclusion, we report that impairment of odr-3 and ife-2 increases lifespan and healthspan in an additive and synergistic manner, respectively, and that this result is not improved by further knocking-down cku-70.
\end{abstract}

\section{INTRODUCTION}

The aging process might be defined by the progressive loss of viability and by an increase in fragility and vulnerability [1, 2]. This in turn, results in a huge health-related cost for the elderly and a dramatic growth in the mortality rate. Understanding the mechanisms underlying aging is one of the major biological and biomedical challenges of our society, and could result in high dividends if the society would gain the capacity to extend lifespan, and more importantly healthspan (i.e. the interval of healthy, productive life years) [3-5]. Although there is still much debate about the molecular causes of aging, the general consensus in the field is that aging is malleable, and studies in model organisms have already shown that aging can be manipulated by both genetic and environmental factors [6-8]. Up until now, more than 2,200 single-gene interventions have been reported to modulate lifespan in model organisms [9]. Most of these genes have been found through genetic interventions, including partial or full loss-offunction mutations, RNA-induced gene silencing, gene over-expression, and genetic polymorphisms, which were reported to promote longevity or cause a premature aging phenotype [9]. More importantly, it has been shown that a large part of these genes play a conserved role as longevity regulators across diverse taxa [10], and some of them even share similar gene 
expression levels in long-lived species [11, 12], overall suggesting that some of the reported longevityassociated interventions could have therapeutic implications even in humans.

The effect on the mean and/or maximum lifespan of the modified organisms ranges from very modest values (5$10 \%$ change) up to very high values, for well-established longevity-associated genes - for example, two-fold for $d a f-2$ in worms [13], six-fold for SIR2 in yeast [14], and even ten-fold for age-1 in worms [15]. Genetic modifications have been identified even in mammals, albeit the observed effects so far seem to be smaller (up to a maximum of 50\%) [9]. These works have significantly increased our knowledge about the genetics of aging and longevity in model organisms, and they should be followed by investigations into the effect of epistatic, or more precisely synergistic gene combinations on lifespan. This aspect, however, has been unfortunately less popular, mainly because the epistasis between longevity-associated genes, and between the pathways they are involved in, is complex and most often non-linear [16, 17], thus requiring much time and resources to be studied. In a recent paper, describing the SynergyAge database, we have defined three types of synergism, applied to the general case of $\mathrm{N}$ genetic interventions: 1) full synergism, in which lifespan values are known for all intermediary strains that contain any combination of the $\mathrm{N}$ interventions and the lifespan change for the n-mutant is greater than the sum of lifespan changes for any two intermediary k-mutant and (N-k)-mutant, 2) simple synergism, in which lifespan values are known for the final strain ( $\mathrm{N}$ interventions) and for all single gene interventions, but not for all intermediary k-mutants, and in which the lifespan effect of the N-gene combination is greater than the sum of all the individual effects, and 3) partially known synergism, in which values are available only for an incrementally built model and for all genetic interventions in an $\mathrm{N}$ sequence an increase in lifespan is observed [18].

The few seminal discoveries regarding longevity synergism generally include the well known IIF/FOXO pathway and the daf-2/daf-16 genes, and have been started in C. elegans [6, 19, 20]. The SynergyAge database reports 62 synergistic combinations of prolongevity interventions that include daf-2. Interestingly, based on SynergyAge data, we did not observe a general correlation between the strength of the longevity effect in WT with those in the long-lived daf2 mutant. For example, RNAi of let-363 did not extend the lifespan of the daf-2(mu150) mutant [21], even though the two genes have the 2nd and 3rd largest increase of lifespan in WT (according to GenAge). sod2 , another important longevity-associated gene, whose deletion leads to a lifespan increase in WT, does not further extend the lifespan of daf-2 mutants [22]. Moreover, three of the top daf-2 enhancers have only a small effect in WT, when kept under same conditions as in the daf-2 background: clk- 1 increases lifespan by only $1.18 \%$ compared to WT at $25^{\circ} \mathrm{C}$ [23] even though at this temperature extends daf-2 lifespan by $205 \%$; rsks-1 increases lifespan of $d a f-2$ by $106 \%$, but only by $20 \%$ in the WT [24]; $d r p-1$, which potentiates the effect of daf-2 by $73 \%$, increases lifespan of WT by only $2 \%$ [25]; clk-2 increases daf-2 effect by $+113 \%$ while in the WT the effect is limited to $68 \%$ [26]. In our study, the genes to be tested were selected based on several bioinformatic criteria (potential of being longevity enhancers for the daf-2 knock-down, genes being part of individual clusters in a cross-database interactome, number of shared KEGG pathways, chromosome positions, etc.), followed by manual curation and evaluation (of scientific literature) for the short-listed gene combinations.

In mammals, the homologues of daf-2 and daf-16 are components of the mammalian insulin and insulin growth factor (IGF) signal transduction cascade (IIS) [27-29]. DAF-2 regulates endocrine responses to food availability, including longevity, dauer formation, and fat metabolism $[13,30,31]$. Mutations that reduce the function of DAF-2 extend lifespan through a mechanism that greatly depends on the activity of DAF-16 [32, 33]. In addition to the central role in integrating signals from insulin/insulin-like pathways, DAF-16 integrates signals from multiple upstream pathways to regulate various biological processes [34]. Due to the increased amount of data on daf-2 and daf-16 mutants, it is extremely appealing to search for genetic interventions that act synergistically amongst themselves, but also with the daf-2 long-lived background. In this study, three such genes have been considered: odr-3, ife-2 and cku-70.

Several sensory neurons are responsible for chemotaxis to volatile attractants found in food, pheromones or noxious odors [27, 35, 36], the nutrient perception by olfactory neurons being partially mediated by the DAF2 pathway [36]. ODR-3, a $G$ alpha protein with similarities to the members of Gi/Go protein family, is expressed in the sensory cilia of olfactory neurons, providing the main stimulatory signals for AWA and AWC sensory neurons $[37,38]$. Ablation of AWA and AWC sensory neurons, as well as loss-of-function mutations in $o d r-3$, extend lifespan through a pathway that depends partially or completely on signaling via DAF-16 [27, 36, 39]. Food restriction can promote an adaptive metabolic response such as mobilization of fat stores through activation of AWC neurons [40], and decreased DAF-2 signaling is known to affect cellular metabolism by promoting the accumulation of lipids in the intestine and hypodermis [30]. All these suggest a 
link between food sensing, metabolic adaptation and longevity. On the other hand, the daf-2(e1370); odr3(n1605) double mutant shows a greater lifespan extension than either of the single mutants and even than their cumulative effects, thus odr-3 and $d a f-2$ could also function through complementary pathways [39].

While the relationship between ROS and longevity is still not completely understood and ROS can have both beneficial or detrimental effects on lifespan, most of the genetic manipulations that decrease ROS lead to an increased lifespan [41]. Like its mammalian orthologue, eIF4E, the $C$. elegans IFE-2 plays an important role in protein synthesis and its inactivation protects against oxidative stress and extends lifespan [42]. Since ife-2 impairment was found to extend the lifespan of longlived mutants such as daf-2, clk-1, eat-2 and let-363, it was suggested that down-regulation of protein synthesis induced by ife-2 deficiency might represent a distinct mechanism by which lifespan is regulated [21, 42]. However, ife-2 inactivation might extend lifespan not only by decreasing the rate of protein synthesis, but also by regulating mitochondrial and peroxisomal metabolism, which in turn, could stabilize the homeostasis of reactive oxygen species and increase cellular accumulation of trehalose [43].

Lastly, CKU-70 is the C. elegans orthologue of KU70, which in mammals participates with KU80 to the DNA repair of double-strand breaks [44]. Downregulation of CKU-70 activity was found to increase sensitivity to genotoxic stress and thermotolerance, thus indicating a conserved role in both DNA repair and stress response $[45,46]$. Although RNA interference (RNAi) of cku-70 increases the lifespan of wild type (WT) animals only in an RNAi sensitized background, the fact that cku-70 knock-down extends the lifespan of daf-2 mutants as well [46] suggests that $c k u-70$ might have an important role in aging.

Since $o d r-3$, ife-2 and $c k u-70$ deficiencies all potentiate the lifespan-extending effects of daf-2 mutants, it is also interesting to find if their mechanisms involve downstream pathways that converge toward common effectors. In this work, we analyzed the effect of combined interventions in odr-3, ife-2 and $c k u-70$, on lifespan and healthspan, starting with L4 age. Since our lifespan and healthspan assays were carried out for all the combinations of the above-mentioned interventions, the use of synergism in the remainder of the paper refers to the "full synergism" definition. Our results show that simultaneous suppression of odr-3 and ife-2 functions additively extends lifespan and synergistically improves healthspan in a daf-16 dependent manner. Knock-down of $c k u-70$ did not confer further benefits to lifespan or motility of $o d r-3$; ife-2 mutants.

\section{RESULTS}

\section{RNA interference of ife-2 but not cku-70 increases lifespan of the long-lived $o d r-3$ mutants}

To find new potential genetic interactions that could extend lifespan, we assessed the effect of a simultaneous depletion of ODR-3, IFE-2 and CKU-70. For this, we used the $o d r-3(n 1605)$ putative null allele [37] and we knocked-down ife- 2 and $c k u-70$ by RNAi. The odr-3(n1605) animals exhibited at $20^{\circ} \mathrm{C}$ an increased mean (26.2\%) and maximum (13.8\%) lifespan compared with WT control animals. A significant mean lifespan extension was previously reported for $o d r-3(n 1605)$ at $25^{\circ} \mathrm{C}$, however the increase was very modest at $20^{\circ} \mathrm{C}$ [39]. Silencing of ife- 2 by RNAi showed an $18.0 \%$ and $20.7 \%$ extension for the mean and maximum lifespan of WT, respectively (Figure 1A and Supplementary Table 1), which are in agreement with previously reported data for both ife-2(ok306) mutants and ife-2 downregulated animals $[21,42]$. In our experiments, the RNAi knockdown of $c k u-70$ in the WT worms produced only a marginal $4.4 \%$ increase for mean lifespan and was even slightly detrimental to maximum lifespan reducing it by $6.9 \%$ (Figure 1B and Supplementary Table 1), which is in agreement with previously reported data [46].

The odr-3(n1605); ife-2(RNAi) mutants exhibited more than $11 \%$ and $18 \%$ increase in mean lifespan compared with the odr-3(n1605) and ife-2(RNAi) single gene interventions, respectively (Table 1). Similarly, the maximum lifespan was also increased by more than $18 \%$ and $11 \%$ (Supplementary Table 1). Overall, compared with the WT controls, the combined odr-3 and ife-2 interventions extended mean and maximum lifespan by $40.3 \%$ and $34.5 \%$, respectively (Figure 1A and Table 1 and Supplementary Table 1). This effect demonstrates an almost additive impact on mean lifespan, i.e. $40.3 \%$ increase compared to $44.2 \%$, the sum of the two individual effects (Table 1). Similarly, the lifespan extension for $o d r-3$; ife-2; $E V(46.1 \%)$, i.e. worms exposed to a 1:1 mixture of HT115 (Empty Vector - EV) bacteria and of ife-2 RNAi clone, was greater than the sum of individual effects $(26.2 \%+$ $11.2 \%$ ), supporting the existence of additive/synergistic mechanisms (Table 1).

Next, we assessed the effect of $c k u-70$ silencing in both odr-3(n1605) mutant animals and ife-2 knock-down animals. We observed that $c k u-70$ knock-down dramatically decreased the extension of mean lifespan conferred by the $o d r-3(n 1605)$ mutation, from $26.2 \%$ to only $13.6 \%$ increase comparative with WT (Figure 1B and Supplementary Table 1). 


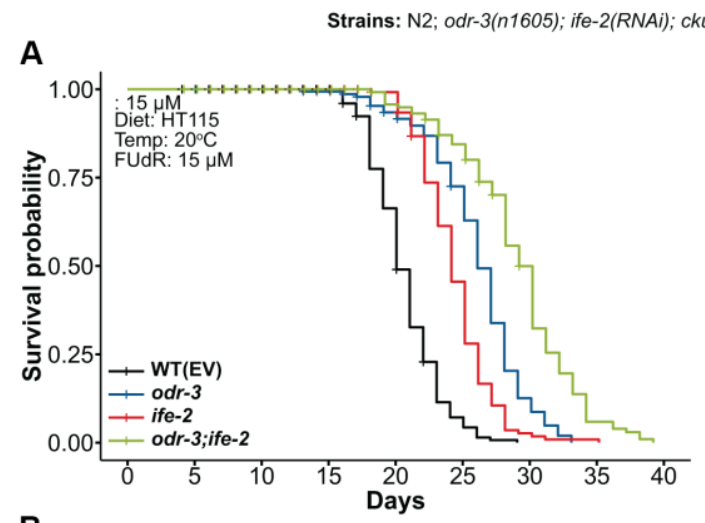

B

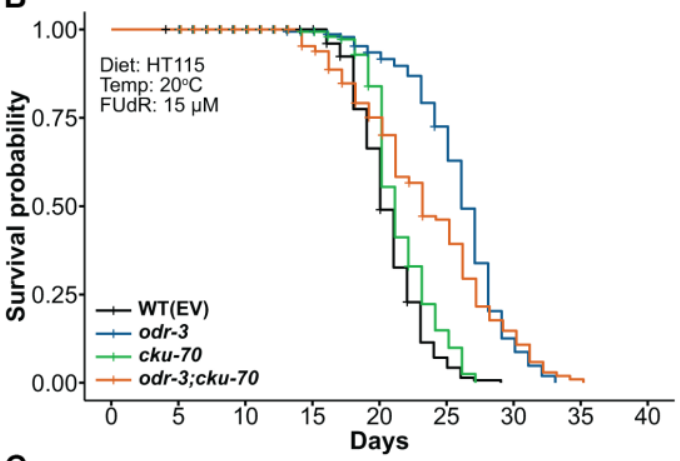

C

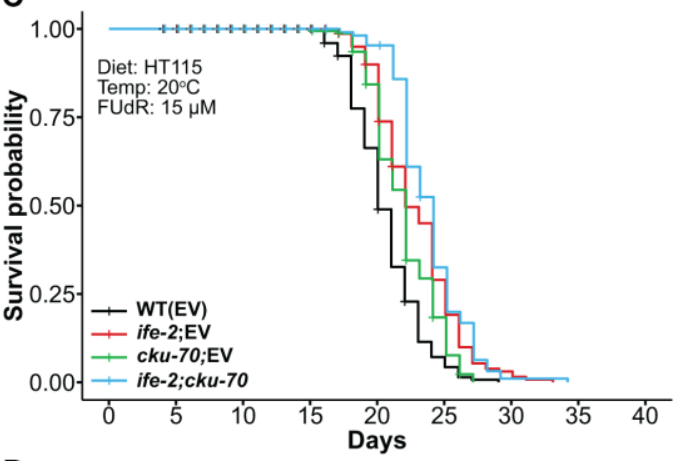

D

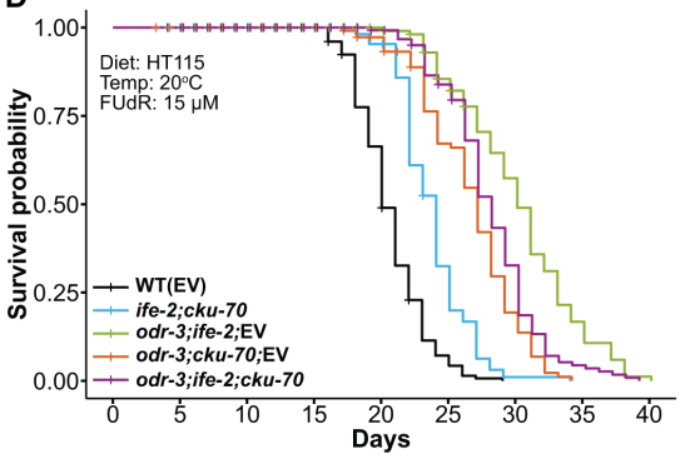

(RNAi); daf-16(mu86)

E

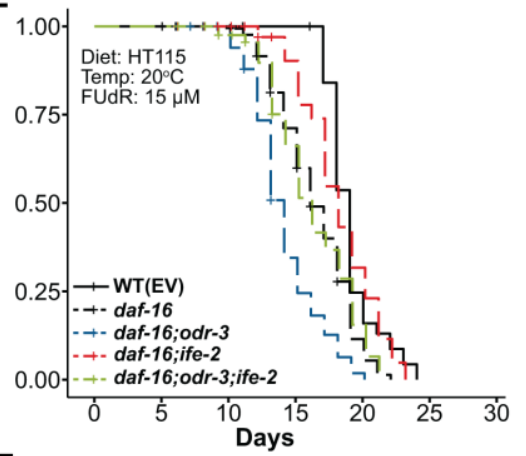

$\mathbf{F}$

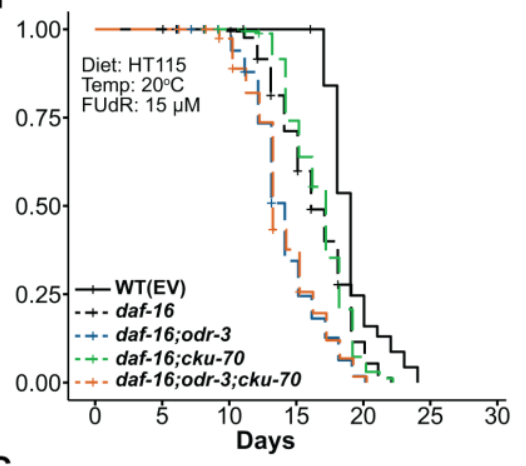

G
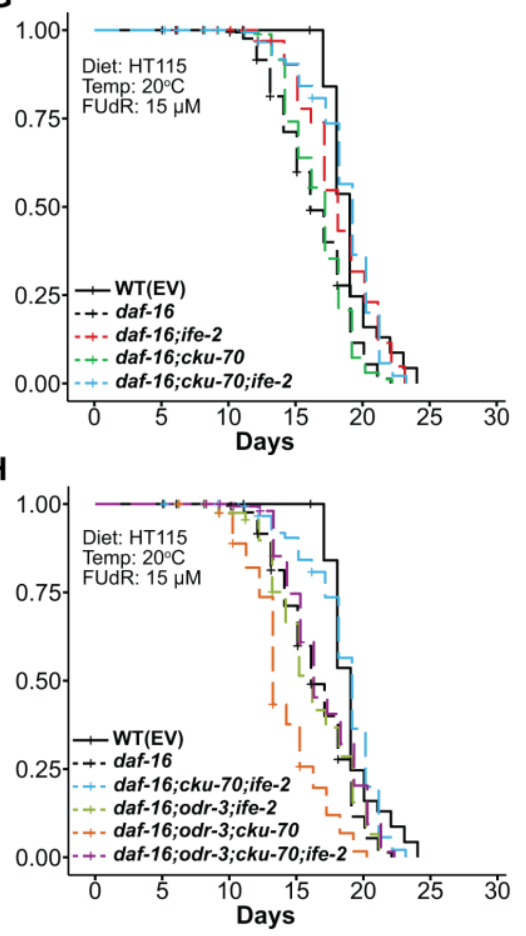

Figure 1. Kaplan-Meier survival curves depicting the effects of combined genetic interventions on odr-3, ife-2 and cku-70 at $20^{\circ}$ C. (A-D) Lifespan comparisons in the WT background (continuous lines). (E-H) Lifespan comparisons in the daf-16(mu86) background (dashed lines). Survival curves represent: (A, E) odr-3(n1605) and ife-2(RNAi) single and double genetic interventions; (B, F) odr-3(n1605) and cku-70(RNAi) single and double genetic interventions; (C, G) ife-2(RNAi) and cku-7O(RNAi) single and double genetic interventions; (D, H) odr3(n1605), ife-2(RNAi) and cku-70(RNAi) double and triple genetic interventions. (C, D) Control in the case of single RNAi knock-downs refers to treatment with a 1:1 mixture of RNAi bacteria and EV bacteria, in order to be comparable to the double RNAi intervention. (A-H) The survival plots in the WT background represent pooled populations from 3 independent experiments, whereas survival plots in the daf$16(m 28)$ background represent pooled populations from 2 independent experiments. odr-3 denotes odr-3(n1605) fed with EV; daf-16 denotes daf-16(mu86) fed with EV; all strains in these experiments were grown on agar plates with E. coli HT115(DE3) and FUdR. 
Table 1. Mean lifespan of $C$. elegans strains with genetic interventions in the odr-3, ife-2 and cku-70.

\begin{tabular}{|c|c|c|c|c|c|c|c|c|c|}
\hline Strain & RNAi* & Mean lifespan days \pm SD & Effect vs control & p-value & Strain & RNAi* & Mean lifespan days \pm SD & Effect vs control & p-value \\
\hline WT & EV & $20.6 \pm 0.2$ & \multicolumn{2}{|c|}{ [control] } & WT & EV & $20.6 \pm 0.2$ & \multicolumn{2}{|c|}{ [control] } \\
\hline$o d r-3$ & & $26.0 \pm 0.3$ & $26.2 \%$ & $<2.0 \mathrm{E}-16$ & $o d r-3$ & & $26.0 \pm 0.3$ & $26.2 \%$ & $<2.00 \mathrm{E}-16$ \\
\hline WT & ife-2 & $24.3 \pm 0.2$ & $18.0 \%$ & $<2.0 \mathrm{E}-16$ & WT & ife- $2 ; E V$ & $22.9 \pm 0.3$ & $11.2 \%$ & $1.00 \mathrm{E}-10$ \\
\hline WT & $c k u-70$ & $21.5 \pm 0.2$ & $4.4 \%$ & $7.00 \mathrm{E}-03$ & WT & $c k u-70 ; E V$ & $21.9 \pm 0.2$ & $6.3 \%$ & $1.00 \mathrm{E}-04$ \\
\hline$o d r-3$ & ife-2 & $28.9 \pm 0.4$ & $40.3 \%$ & $<2.0 \mathrm{E}-16$ & $o d r-3$ & ife- $2 ; E V$ & $30.1 \pm 0.5$ & $46.1 \%$ & $<2.00 \mathrm{E}-16$ \\
\hline$o d r-3$ & $c k u-70$ & $23.4 \pm 0.5$ & $13.6 \%$ & $4.00 \mathrm{E}-11$ & $o d r-3$ & $c k u-70 ; E V$ & $26.5 \pm 0.4$ & $28.6 \%$ & $<2.00 \mathrm{E}-16$ \\
\hline
\end{tabular}

*ife-2 denotes animals fed only with ife-2 RNAi bacteria, whereas ife-2;EV denotes animals fed with a mixture of ife2RNAi/RNAi(EV). Similar for $c k u-70$. odr-3 denotes animals fed with RNAi(EV).

The simultaneous knock-down of ife-2; $c k u-70$ by RNAi was performed by co-feeding worms with a mixture of the two RNAi bacterial clones. As such, for an appropriate comparison, the survival curves of double knock-down worms (which are presumably exposed to about half dsRNA for each gene) have been compared with those of single knock-down worms exposed to the same concentration of dsRNA for each of the corresponding genes (concentrations obtained by co-feeding the worms with the target RNAi clone and the control RNAi(EV) in a 1:1 ratio). In general, we obtained very small differences between the lifespan of worms fed only with the RNAi clone and worms fed with the mixture of RNAi clone / RNAi(EV) (Supplementary Figure 1A-1C), with the only notable difference being for $o d r-3$; $c k u-70$ for which the mix (and hence lower concentration of $c k u-70$ RNAi bacteria) did not show a pronounced lifespan reduction (Supplementary Figure 1D).

In our assays, the lifespan of the double knock-down worms ife-2; cku-70 was $15 \%$ longer than that of WT animals $(\mathrm{p}=3.0 \mathrm{E}-16)$, with a small increase compared to each of the single knock-down worms $(3.5 \%$ and $8.22 \%$ longer lived than the ife-2; EV and the $c k u-70$; EV animals, respectively) (Figure 1C and Supplementary Table 1). It should however be noted that these changes are very modest and that a slightly larger lifespan increase was obtained when worms were exposed to ife-2 RNAi alone, without EV mixing (18\% compared to WT). Moreover, $c k u-70$ knock-down had a negative effect on the lifespan of $o d r-3(n 1605)$ mutants treated with ife-2 RNAi, decreasing the mean lifespan extension from $46.1 \%$ to $35.4 \%$ (Figure 1D and Supplementary Table 1).

The extended longevity of odr-3; ife-2 double
intervention might be independent of DAF-16

The FOXO family transcription factor DAF-16 is a transducer of many pro-longevity signaling pathways
[47], thus it was natural to inquire to what extent the longevity of $o d r-3$; ife-2 double inactivated animals require DAF-16. To answer this, we used the null daf$16(m u 86)$ allele $[32,48]$ that affects coding of all DAF16 isoforms, to generate daf-16(mu86); odr-3(n1605) double mutants, and carried out RNAi silencing assays for ife-2 and cku-70 in this strain.

We observed that the lifespan extension induced by the odr-3(n1605) mutation was not only suppressed by the daf-16(mu86) mutation, but also that the lifespan of the daf-16(mu86); odr-3(n1605) double mutants were even shorter than the lifespan of the daf16 mutants alone (Figure 1E; mean and maximum lifespan decreased by $14.6 \%$ and $9.1 \%$ compared to daf-16; mean and maximum lifespan decreased by $26.3 \%$ and $16.7 \%$ compared with WT). A slight decrease of daf-16(mu86) lifespan induced by the odr3(n1605) mutation was also previously reported [39]. Silencing of ife-2 on the other hand, extended the lifespan of daf-16 mutants (Figure 1E; mean lifespan $9.8 \%$ greater), although the mean and maximum lifespan were not completely reverted to the lifespan values of the WT (mean and maximum lifespan 5.3\% and $4.2 \%$ lower than WT, respectively). The lifespan of the triple daf-16; odr-3; ife-2 inactivated animals did not significantly differ from daf-16 single mutants. This could be partly explained by the fact that DAF-16 is one of the main transducers of signaling pathways modulated by ODR-3 and IFE-2 activity. To clarify this aspect we examined the nuclear translocation of DAF-16::GFP in $o d r$ 3(n1605), ife-2(RNAi) and odr-3(n1605); ife-2(RNAi) animals, respectively (Supplementary Figure 2). Whereas odr-3(n1605) animals showed weak DAF$16::$ GFP nuclear accumulation in posterior intestinal cells, suggesting that ODR-3 could affect longevity partially through DAF-16 pathway, we did not observe consistent nuclear accumulation of DAF$16:: \mathrm{GFP}$ in ife-2(RNAi) and odr-3(n1605); ife$2(R N A i)$ animals. Therefore, ODR-3 and IFE-2 could 
affect lifespan by mediating parallel signaling pathways, which in the daf-16 background have antagonistic effects - odr-3 further decreasing lifespan and ife-2 partially increasing the daf-16(mu86) lifespan.

In contrast to a previous study that reported a slight decrease of daf-16( $\mathrm{m} 26)$ lifespan by $c k u-70$ knockdown at $25^{\circ} \mathrm{C}$ [46], in our experiments the lifespan of daf-16; cku-70 at $20^{\circ} \mathrm{C}$ was similar to that of daf-16 single mutants, and cku-70 knock-down did not significantly influence the lifespan of daf-16; odr3 , nor of daf-16; ife-2 mutants (Figure 1F, 1G). The quadruple daf-16; odr-3; ife-2; cku-70 mutants exhibited a lifespan similar to that of daf-16 single mutants (Figure 1H).

Overall, our results show that simultaneous inactivation of $o d r-3$ and ife-2 produce an additive lifespan effect, while the additional cku-70 knock-down does not extend lifespan further (Figure 2). Since the lifespan effects observed are different in the daf-16 background (Figure 2), it is possible that the mechanisms through which odr-3; ife-2 animals achieve lifespan extension overlap with the pleiotropic mechanisms determined by daf-16 (Figure 2).

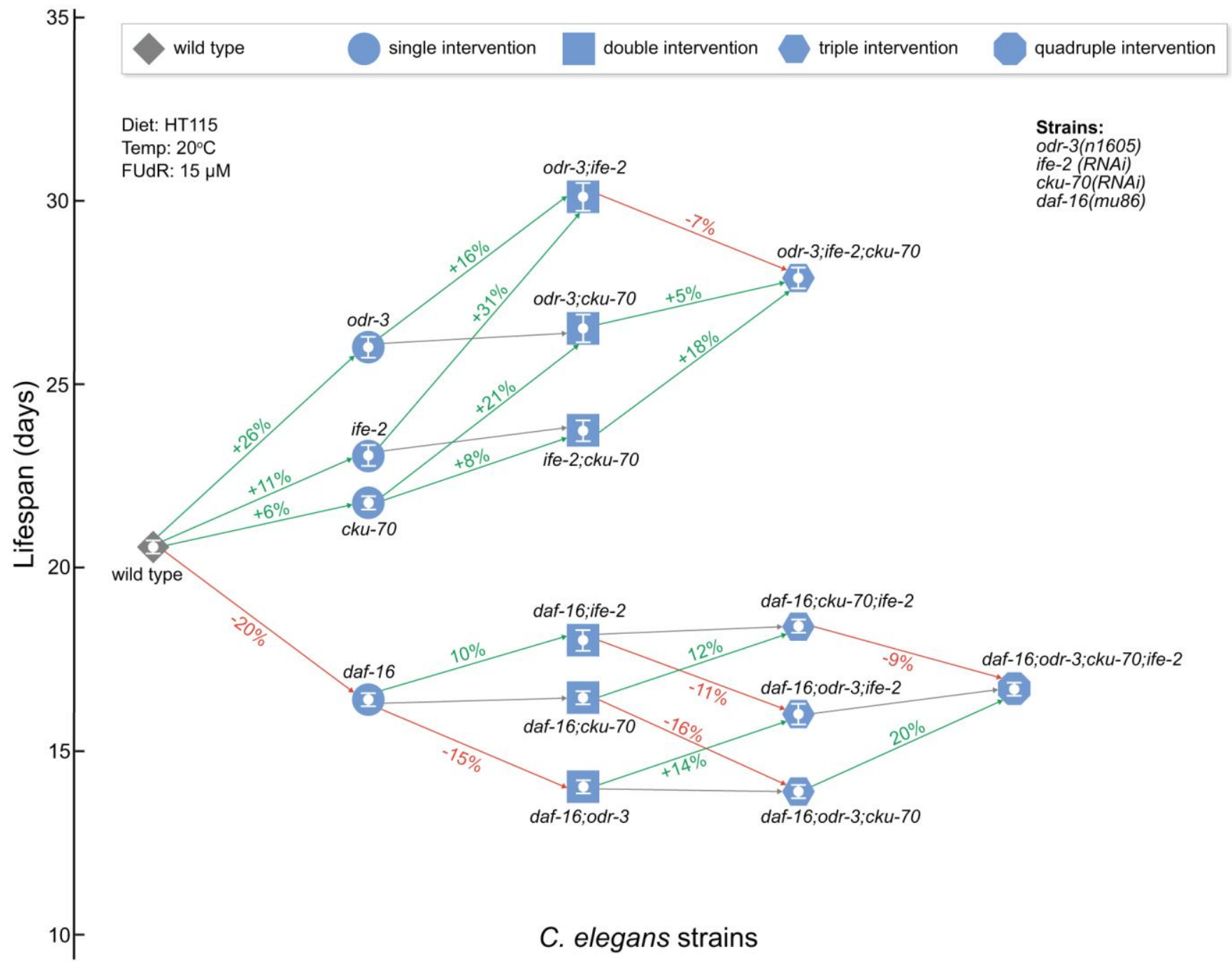

Figure 2. Network schematic representation of the strains analyzed in this study and of the effects of each genetic intervention. Nodes represent the strains as follows: diamond for WT, circle for single gene interventions, square for double gene interventions, hexagon for triple gene interventions, and octagon for quadruple gene interventions. Nodes are positioned on the vertical axis according to their respective mean lifespan. Edges between worm strains are colored depending on the gain (or loss) in lifespan extension: increase (green), decrease (red) and small or non-significant change (gray). The extent of the change is included on the edge as a percentage increase/decrease between the origin and destination nodes of the edge. odr-3 and daf-16 denote mutants containing the odr-3(n1605) and daf-16(mu86) mutations; ife-2 and cku-70 denote animals in which these genes were modulated by RNAi bacteria. The white bars inside of the nodes indicate the mean \pm SEM. 
The ife-2(ok306) mutation also extends the lifespan of odr-3(n1605) mutant animals

One question is whether the ife-2(ok306) deletion mutation will produce similar effects as the ife-2(RNAi) in the odr-3(n1605) background. To answer this, we also generated the double odr-3(n1605); ife-2(ok306) and triple daf-16(mu86); odr-3(n1605); ife-2(ok306) mutants and conducted further lifespan assays. The mutant worms were cultured at the same temperature $\left(20^{\circ} \mathrm{C}\right)$ as in the RNAi experiments and were fed OP50 bacteria. We observed similar trends (Figure 3A), i.e. a $13.6 \%$ and $35.7 \%$ increase in mean and maximum lifespan for the $o d r-3$; ife-2 double mutant, compared to wild type, and an additive effect of the single mutations (the $13.6 \%$ increase in mean lifespan was comparable with the sum of the individual genetic effects of $o d r-3$ and ife-2 mutations: $7.5 \%$ and $4.5 \%$ ). It was however noticeable that in this experiment the impact of the double mutation on lifespan was smaller than in the case of ife-2(RNAi), which used an HT115 diet.

Since FUdR could cause an artefactual effect on the longevity of some mutants [49-51], we also conducted longevity experiments in the absence of FUdR. Similar to the previous results, we also observed an increased average lifespan for all strains even in the absence of FUdR (Figure 3B, odr-3:+8.2\%, ife $-2:+8.6 \%$ and $o d r-$ 3 ;ife-2:+14.3\%).

Next, we investigated the effect of the $o d r-3(n 1605)$ and ife-2(ok306) mutations in the daf-16(mu86) background. Similar to the RNAi experiments, the ok306 extended the lifespan of daf-16 single mutants (Figure 3C, 6.25\% increase), while the double daf-16(mu86); odr-3(n1605) and triple daf-16(mu86); odr-3(n1605); ife-2(ok306) mutants showed very similar lifespans as that of the daf16 (Figure 3C).

\section{The odr-3; ife-2 impaired animals display increased motility and pharyngeal pumping}

Our finding that RNAi impairment of ife-2 in odr3(n1605) animals increased lifespan prompted us to investigate the effect of their inactivation on healthspan. To assess healthspan, we focused on the evaluation of pharyngeal pumping and body movement. In $C$. elegans, these two physiological processes decrease with ageing, correlate between themselves and with other age-related declining properties, and ultimately can predict lifespan and healthy life [52].

In our experiments, up to late adulthood, individual or joint interventions in $o d r-3$ and ife- 2 did not produce obvious pathological changes in the phenotype, indicating that the effect on locomotion was caused by physiological age-related changes, rather than a specific disease. As such, the observed motility status, carried out along the lifespan assay, can be viewed as a measure of the healthspan of the population. To quantify this, we classified individuals in three motion stages based on their ability to move. Stage A (healthy, fully mobile worms) included animals in a physiological state that could move without any impediment, stage B (impaired worms) included animals with diminished locomotion, whereas stage $\mathrm{C}$ (frail worms) included animals found in a frailty state. Animals were scored daily and associated with one of the motion stages. The distribution of stages for each strain is presented graphically in Figure 4A-4D (for
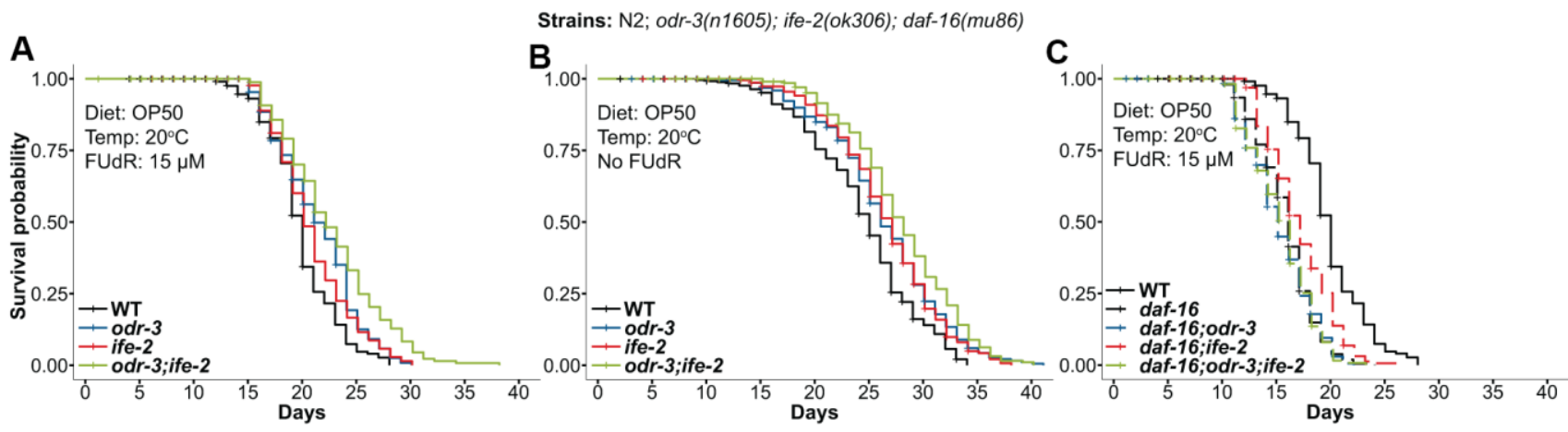

Figure 3. Kaplan-Meier survival curves for animals containing the odr-3(n1605) and ife-2(ok306) mutations. (A) odr-3(n1605); ife-2(ok306) single and double mutants, cultivated in the presence of FUdR. (B) odr-3(n1605); ife-2(ok306) single and double mutants, cultured without FUdR. (C) Lifespan comparisons for odr-3 and ife-2 in the daf-16(mu86) background. (A-C) Dashed lines are used for odr3 (n1605) and ife-2(ok306) mutants tested in the daf16(mu86) genetic background, while continuous lines are used for WT or single/double odr-3 and ife-2 mutants tested in the WT background. All cohorts were fed OP50 and kept at $20^{\circ} \mathrm{C}$. All lifespan values can be viewed in the Supplementary Table 1. 
A

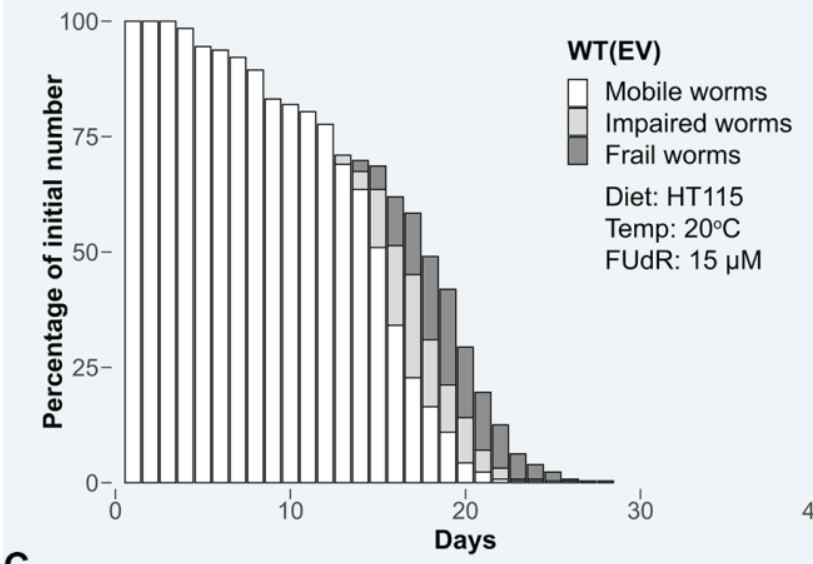

C

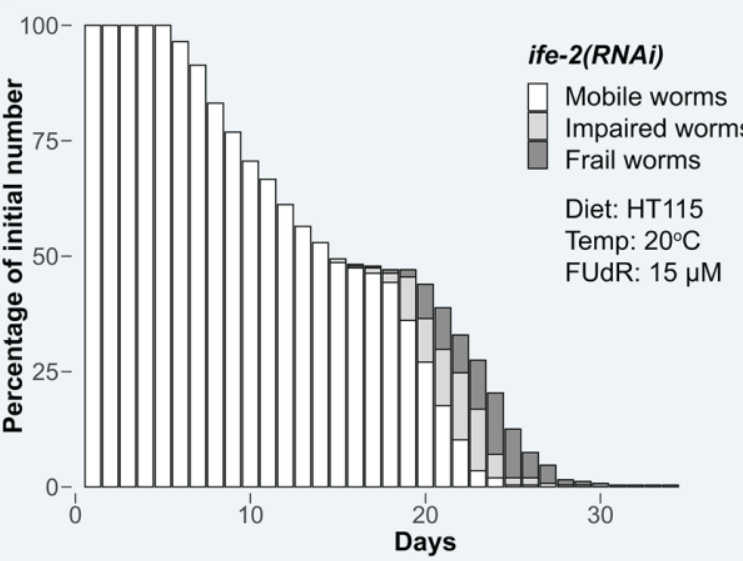

E

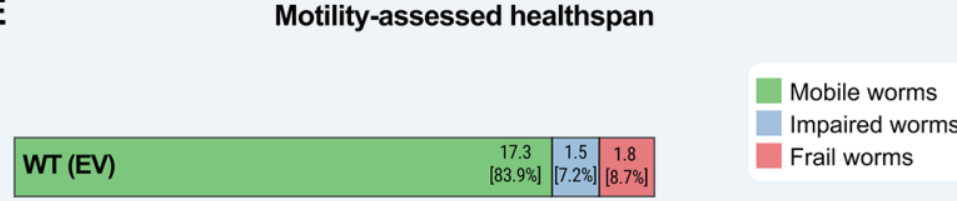

B

D

\begin{tabular}{|c|c|}
\hline ife-2(RNAi) & \begin{tabular}{|c|c|c|}
21.0 & 1.6 & 1.7 \\
{$[86.4 \%$} & {$[6.5 \% \mid$} & {$[6.9 \% \mid$} \\
\end{tabular} \\
\hline
\end{tabular}

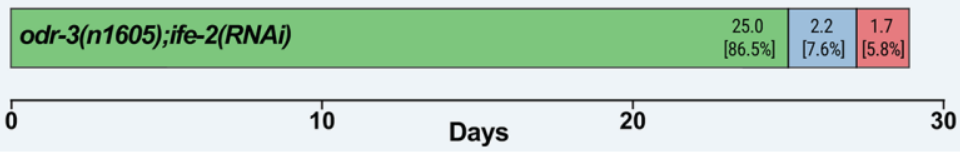
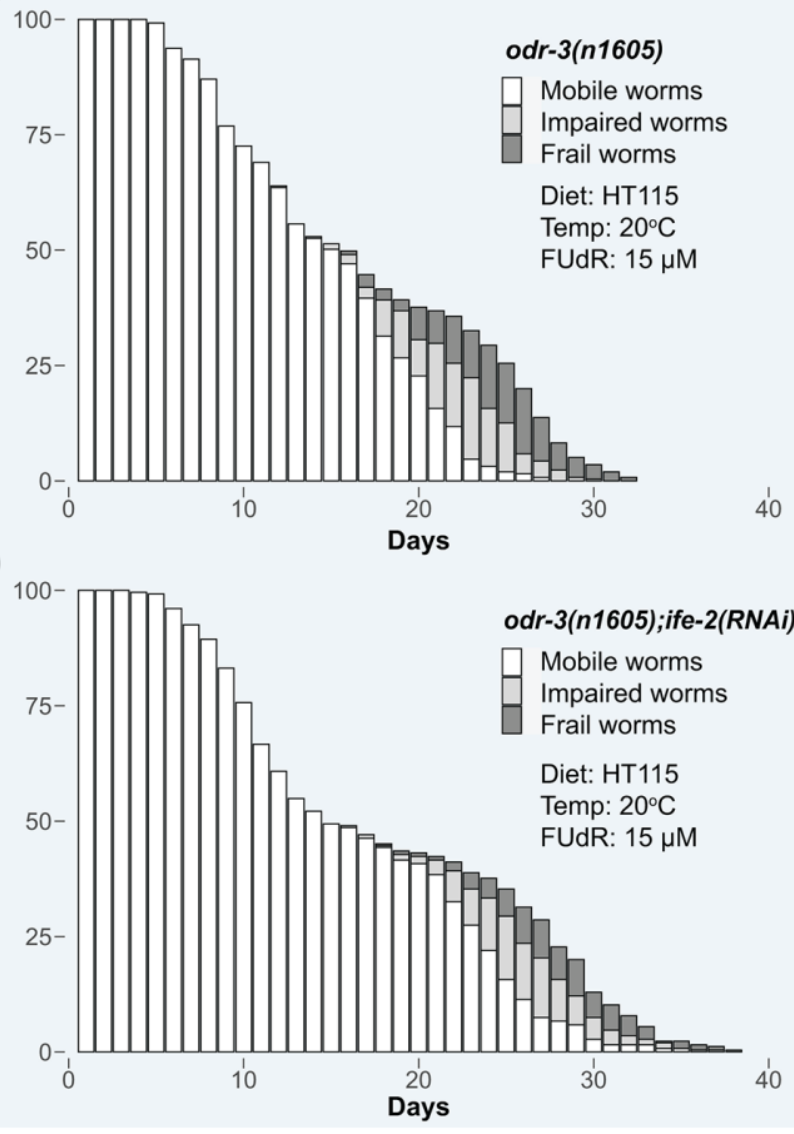

F Pharyngeal pumping-assessed healthspan

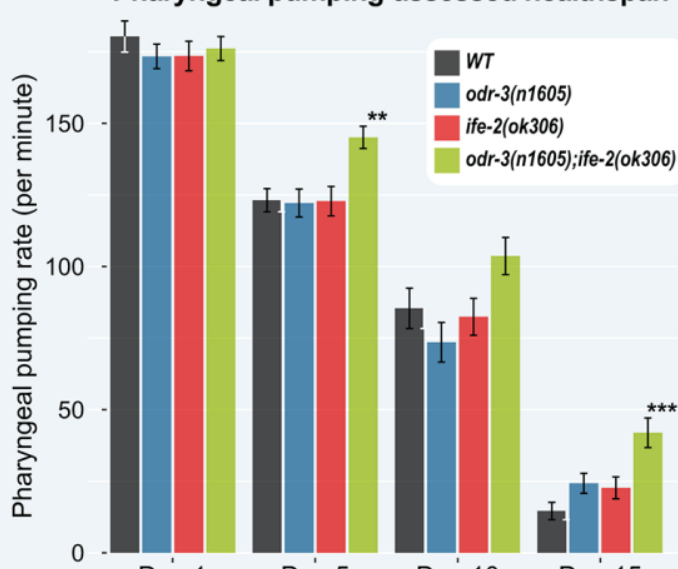

Figure 4. Healthspan of combined genetic interventions on odr-3 and ife-2 at $20^{\circ} \mathrm{C}$. (A-D) Bar chart representation of motilityassessed healthspan illustrating the fraction of each category upon daily monitorization. Worms are grouped into three categories: mobile (white), impaired (light gray) and frail (dark gray). Dead and censored animals were subtracted from these analyses. (E) Mean number of days in each motility state throughout lifespan. The mean time spent in the impaired state is computed as the difference between the mean time spent as mobile or impaired, and the mean time spent in the mobile state. The mean time spent in the frail state is computed as the difference between the mean lifespan and mean time spent as mobile or impaired. The values within brackets represent the distribution of motion stages during the lifespan. (A-E) WT(EV) and odr-3 denote worms fed with RNAi(EV). (C, D) ife-2 and odr-3; ife-2 denote worms fed with ife-2 RNAi bacteria. (F) The pharyngeal pumping rate (average number of contractions per minute) of WT, odr-3(n1605), ife-2(ok306) and odr-3(n1605); ife-2(ok306) mutants were recorded on days 1, 5, 10 and 15 post-L4 moult. odr-3(n1605); ife-2(ok306) worms show a significantly slower decline of pharyngeal pumping with age, compared to WT. For simplicity, only significant differences among groups are indicated (one way ANOVA with Dunnett's test); ${ }^{* *}$ denotes $p<0.01 ; * *$ denotes $p<0.001$. 
motility data of $o d r-3(n 1605)$; ife-2(ok306) mutants, see Supplementary Figure 3A-3H; for the daf-16(mu86); odr-3(n1605); ife-2(ok306) mutants see Supplementary Figure 3I-3L).

Considering the average healthspan of worms in stage A, we observed a similar trend to lifespan. Simultaneous inactivation of $o d r-3$ and ife-2 produced a synergistic healthspan effect, while the additional cku70 knock-down does not extend healthspan further (Supplementary Figure 4).

Using the Kaplan-Meier method to estimate the fraction of mobile worms at each observation point, plotted against time, the average number of days the worms spent in each state was computed (Figure 4E and Supplementary Table 2). This allowed us to model the transitions from the healthy to impaired or frail (see Materials and methods), thus, determining if a change in the locomotion status was induced by the genetic interventions and whether in addition to lifespan, the ratio between healthspan and lifespan was also changed.

The WT animals spent on average 17.3 days in the mobile stage, which represent $84 \%$ of their mean lifetime, 1.5 days (7.3\%) in an impaired stage and 1.8 days $(8.7 \%)$ in the frailty stage. The odr-3 mutants remained mobile longer than WT animals (on average 20.8 days), however relative to their mean lifespan, they were fully mobile only for $80.0 \%$ of their lifetime, thus exhibiting a proportional (or greater) lifespan fraction in which they were impaired or frail (2.6 days, $10.0 \%$, for both). ife-2 RNAi treated animals remained mobile on average more days (21.0 days, $86.4 \%$ ) and exhibited a similar number of impaired (1.6 days, 6.6\%) and frail days (1.7 days, $7.0 \%$ ) (Supplementary Table 2).

For the $o d r-3$; ife-2 animals, the longest-lived strain in our study, a corresponding increase of days with full (25.0 days, $86.5 \%)$ and impaired motility (2.2 days, $7.6 \%$ ) was observed, while the number of days of frailty remained similar as for WT animals (1.7 days, 5.9\%).

Next, we compared each fraction of being fully mobile with the corresponding fraction in the WT animals to find if the genetic interventions indeed conferred significant benefits for the quality of life, i.e. increasing the fraction of time spent in a mobile state and decreasing the impaired and frailty fractions. By doing this, we found that although the odr-3 mutants had extended longevity, this was not associated with a motility-based health benefit since the lifespan fraction in which worms lived as fully mobile actually decreased slightly by $4.8 \%$ (Supplementary Table 2), whereas the fraction of time spent in both the impaired and frailty stages increased by almost $40 \%$. By contrast, ife-2 RNAi treated animals stayed mobile for a similar lifespan fraction as WT animals (3\% longer), but they spent much less time as impaired or frail (these fractions were 9.6\% and 19.5\%, respectively, smaller than those for WT). Compared to WT control animals, silencing of ife-2 in odr-3 worms did not affect the mean lifespan fraction spent in the mobile and impaired stages, but decreased the lifespan fraction for the frailty period by more than $30 \%$.

To assess the significance of the changes in motility status, the Kaplan-Meier curves modeling the transitions from mobile to impaired and frailed were used and the relevant comparisons are included in the Supplementary Figure 5C. Overall, our findings show that the ife-2 RNAi treatment and the double intervention odr-3(n1605); ife-2(RNAi) have a beneficial effect on motility-assessed healthspan, significantly increasing the period of full motility (Supplementary Figure 5C; $p<2.0 \mathrm{E}-16$ ) and exhibiting a decrease in the decrepit period of life. Similar data for the odr3(n1605); ife-2(ok306) double mutant can be seen in Supplementary Figure 5D, 5E and for the daf16(mu86); odr-3(n1605); ife-2(ok306) triple mutant in Supplementary Figure 5F.

To further determine whether healthspan is affected, we analyzed the decline of pharyngeal pumping with age, a process controlled by the cardiac-like pharyngeal muscle. As seen in Figure 4F, the number of pharyngeal movements strongly decreases with age in all cohorts. The changes for both single mutants, odr-3(n1605) and ife-2(ok306), are similar to those in the WT (no statistically significant difference observed when comparing to WT, at each day; one-way ANOVA). The odr-3(n1605); ife-2(ok306) double mutant showed a slower decline in pharyngeal contractions and a higher rate of pumping compared to WT. The improved healthspan was observed starting from day 5 , when the average number of contractions was $17.8 \%$ higher than for WT $(\mathrm{p}=0.002)$, and slightly increased, at day 10 being $21.4 \%$ higher $(\mathrm{p}=0.14 ; \mathrm{ns})$. At day 15 , the odr3;ife-2 animals still showed approximately 42 contractions per minute (compared to 15 contractions per minute in the WT), a $187 \%$ increase (p <E-04). On day 15 , the odr-3(n1605) and ife-2(ok306) mutations showed a synergistic effect on the pharyngeal pumping, the increase observed in the double mutant being higher than the sum of the individual effects $(187 \%$ increase compared to $66 \%+55 \%$ increase for single mutants), corresponding to a fully synergic effect [18].

\section{The odr-3; ife-2 double mutant displays an increased resistance to stress}

Resistance to stress declines with age after early adulthood [53], and the loss of protein homeostasis 
together with the failure to activate cellular stress responses are among the earliest aging marks [54]. Elevated temperatures perturb the protein homeostasis due to accumulation of defective proteins, whereas production of reactive oxygen species (ROS) and $\mathrm{H}_{2} \mathrm{O}_{2}$ increase global cellular damage. Both insults activate the cellular stress responses, aiming to improve cellular fitness and organismal recovery. To find if the double intervention in odr-3 and ife-2 perturbs the stress response mechanisms, we monitored survival upon exposure to oxidative stress and acute heat stress. We induced oxidative stress by treatment with either paraquat, which generates reactive oxygen species, or $\mathrm{NaN}_{3}$, which generates $\mathrm{H}_{2} \mathrm{O}_{2}$. Both treatments dramatically reduce survival of WT animals by more than $50 \%$ (Figure 5A, 5B). In contrast, mutation in the $o d r-3$ increases survival of the animals treated with both paraquat and $\mathrm{NaN}_{3}$ compared with treated WT, although not statistically significant for $\mathrm{NaN}_{3}$. ife-2 mutants exhibited an increased survival upon both treatments, as previously reported [42]. The odr-3; ife2 double mutants were less sensitive to ROS and $\mathrm{H}_{2} \mathrm{O}_{2}$ toxicity than WT animals, but they did not show an additive effect when compared with single mutants (Figure 5A, 5B).

Exposure to $35^{\circ} \mathrm{C}$ for $4 \mathrm{~h}$ decreases the survival rate of WT animals by more than $50 \%$ (Figure $5 \mathrm{C}$ ). Whereas the heat shock slightly increased the survival of $o d r-3$ mutants, compared with WT (not statistically significant), it did not also affect the survival of ife-2 mutants or odr-3; ife-2 double mutants (Figure 5C), overall indicating that impairment of $o d r-3$ and/or ife-2 do not affect the heat stress response.
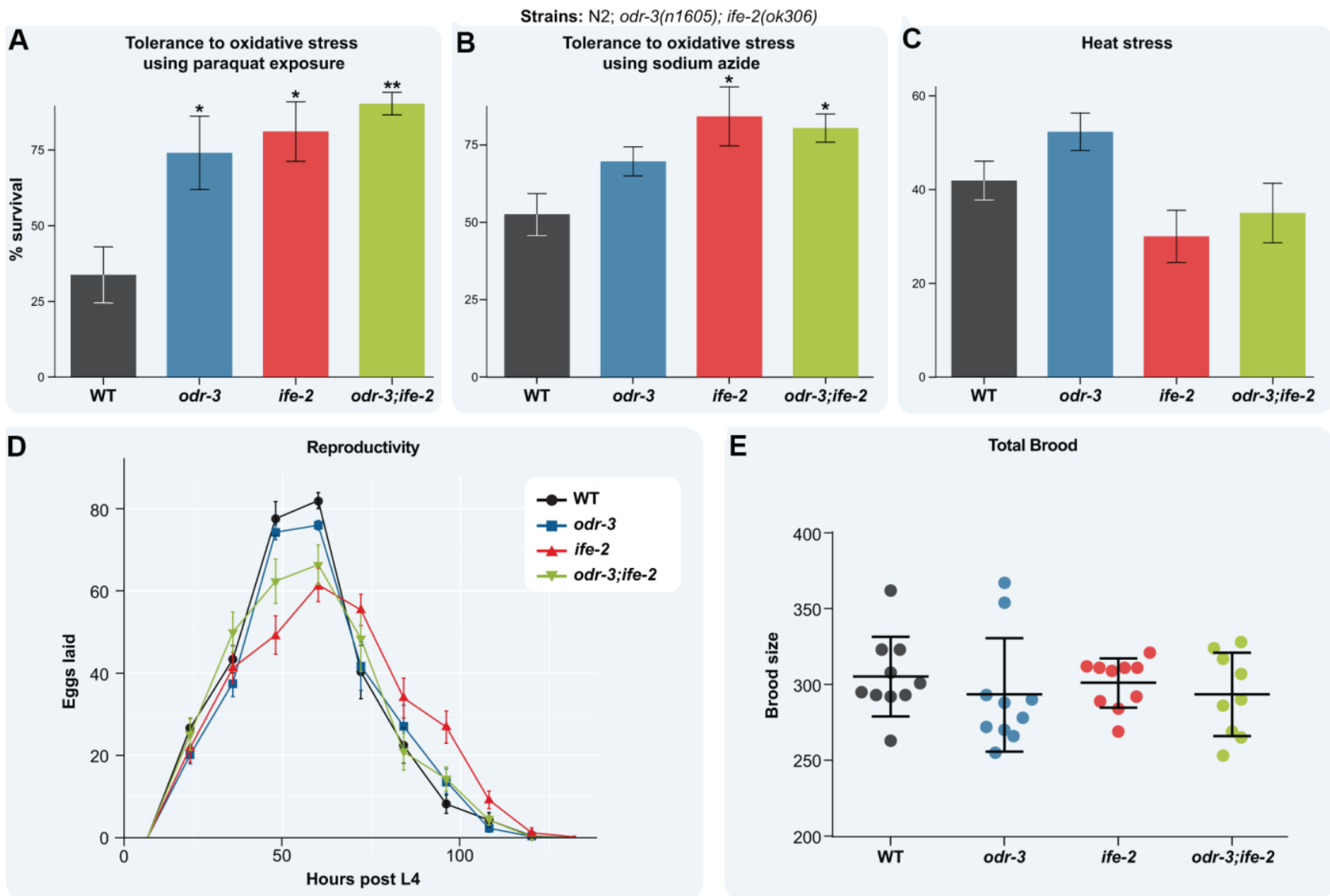

E

Total Brood

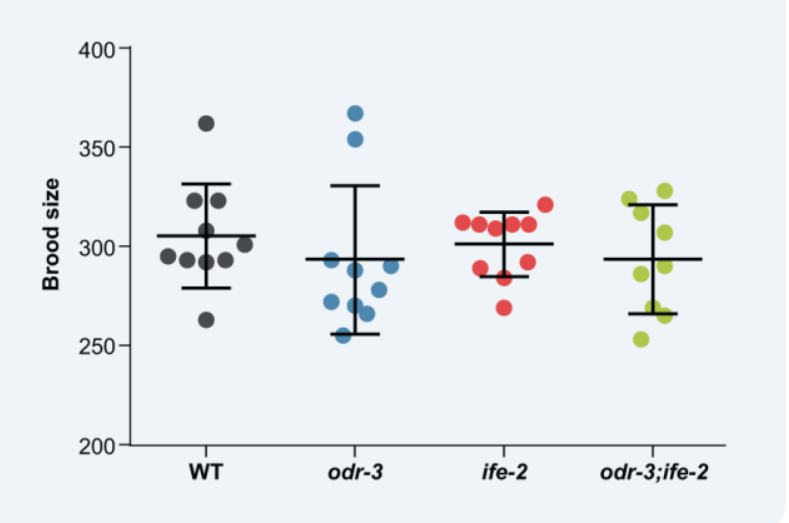

Figure 5. Loss of odr-3 and ife-2 activity enhances oxidative stress tolerance. (A) Survival fraction of the indicated L4 larvae upon 5 days exposure to $0.2 \mathrm{M}$ paraquat (the experiment was repeated independently three times). (B) Survival fraction of the indicated strains upon 1 hour treatment with $0.5 \mathrm{M} \mathrm{NaN3}$ (the experiment was repeated independently four times). (C) Survival fraction of the indicated strains upon $4 \mathrm{~h}$ heat stress at $35^{\circ} \mathrm{C}$. Each strain was scored on three replicate plates and the experiment was repeated independently four times. (D) Egg-laying rate of the indicated strains. The average number of eggs laid by each strain was determined by transferring worms to new $E$. coli plates every 12 hours from L4 stage. (E) Brood size of the indicated strains at $20^{\circ} \mathrm{C}$. Each point represents the total brood of one hermaphrodite. (A-E) Bars indicate the mean \pm SEM. For simplicity, only significant differences among groups are indicated (one way ANOVA with Dunnett's test); * denotes $p<0.05 ; * *$ denotes $p<0.01$. 
Some genetic or non-genetic interventions that extend lifespan also reduce fecundity, implying a trade-off between longevity and reproduction [55-57]. We did not observe such an effect for $o d r-3$ and ife-2 single and double mutants (Figure 5D). Thus, the reproductive period and the age of the peak egg-laying rate of single and double mutants coincided with that of WT animals (Figure 5D). Moreover, the brood size of the mutants is similar to that of WT animals (Figure 5E). In conclusion, simultaneous depletion of odr-3 and ife-2 extends lifespan without affecting fecundity, promotes muscle activity and maintains activation of stress response mechanisms, consistent with increased health.

\section{DISCUSSION}

Longevity is regulated by a combination of genetic and non-genetic factors, such as environmental interactions and lifestyle. The identification of genetic mutations that extend lifespan in model organisms has shown that longevity is mainly regulated by a complex interplay between many signaling pathways that affect cellular functions as diverse as nutrient sensing, genome stability, mitochondria fitness, organelle proteostasis, intercellular communication, transcription, proliferation and cellular regeneration [58,59]. Previously, we have successfully used network-based approaches, building upon the list of known longevity-associated genes hosted in the GenAge database [9], to predict novel genetic or drug interventions that extend lifespan [60, 61]. However, due to the existence of complex and intricate interactions between hundreds of longevityassociated genes [62-64], the lifespan modulation obtained with these methods was limited by how much a single gene can influence longevity. While combined genetic interventions that modulate longevity via parallel pathways or drugs targeting multiple evolutionary conserved aging pathways have been shown in many instances to extend lifespan [65], the number of gene combinations tested so far has not been very high [18]. Here, we assessed the effects on both lifespan and healthspan, given by the simultaneous inactivation of three genes: odr-3, ife-2 and $c k u-70$. By reporting on the synergy of the pro-longevity effects of IFE-2 and ODR-3, and at the same time on the lack of synergy between CKU-70 and the above two genes, we hope that the current work will add to the accumulating data on longevity-related gene combinations, which could be used in future predictions of complex, multigene interventions.

According to the SynergyAge database http://synergyage.info/ [18], the above-mentioned genetic interventions were among the most promising in terms of lifespan extension, when combined with IISdefective daf-2 mutants $[39,42,46]$, a highly desirable property. SynergyAge hosts 133 unique synergistic interactions, involving 108 genes and of these, 62 gene combinations include daf-2. Much less combinations are antagonistic (32 gene combinations) while for 156 gene combinations the effect is somewhere between the two individual effects. Although this summary does not provide information on the number of negative results (which would probably be classified in one of the last 2 categories) it gives a sense of the scarcity of synergistic interactions discovered so far - considering the total number of potential gene combinations that could encompass for example the 889 worm longevityassociated genes from GenAge [9] (even for two gene combinations).

In C. elegans, ODR-3, IFE-2 and CKU-70 have different functions, and although inactivation of each of them extends lifespan, the mechanisms by which this occurs are at least partially different from each other. In our study, the lifespan and healthspan assays were carried out for all these 3 genes and their combinations. As a result, we obtained a clear perspective of the lifespan and healthspan changes from all worm strains, which is represented in Figure 2 and Supplementary Figure 4, respectively. Using this representation, it can be easily observed that the odr-3; ife-2 double inactivation leads to an additive increase (i.e. the effect of the joint interventions is equal to the sum of the individual effects) in lifespan compared with single gene inactivations (Figure 2), as well as a synergistic effect on healthspan (Supplementary Figure 4). On the other hand, $c k u-70$ down regulation does not significantly affect the lifespan of ife-2 mutants (Figure 1C) and is detrimental to the long-lived odr-3 and $o d r$ 3; ife-2 strains (Figure 1B, 1D). This detrimental effect is not seen in the daf-16 mutants, where the loss of both $o d r-3$ and daf-16 seems to be dominant compared to the influence of $c k u-70$ downregulation (Figure 1F). It is difficult to explain the effect of $c k u-70$ down regulation on $o d r-3$ and $o d r-3$; ife-2 only through its function in the DNA repair process. In addition to its role in DNA repair, the conserved $\mathrm{Ku}$ heterodimer was found to participate in other cellular processes such as transcriptional regulation, apoptosis, DNA replication, RNA metabolism and other $[66,67]$. Therefore, a more complex interaction between $c k u-70, o d r-3$ and ife-2 might exist.

In our experiments we double inactivated ife-2 and $c k u$ 70 by RNAi. This raises the possibility that the knockdown efficiency of one or both genes could be reduced to half. For this reason, for an appropriate comparison with single RNAi down regulation we exposed the worms to a half concentration of dsRNA by mixing RNAi clone with EV clone. Unfortunately, we could not verify by quantitative PCR the efficiency of 
ife-2 RNAi down regulation, which gave us the most notable effect, because the DNA fragment used for expression of dsRNA from L4440 vector encompasses the full gene (ORF and UTR) and due to the uptake of dsRNA in the cells, the endogenous ife-2 mRNA cannot be distinguished and targeted for PCR amplification. However, knock down of ife-2 RNAi; EV increased the lifespan of $o d r-3$ mutants, suggesting efficient down regulation even with half concentration of ife-2 dsRNA. We have to point out that in the case of double RNAi there is a possibility that worms are not equally exposed to both dsRNA, and down regulation of one or both genes might be less efficient. Hence, although single downregulation of $c k u-70 \mathrm{RNAi}$ gave only a very modest increase of lifespan, the effect of double inactivation ife-2(RNAi; cku-70(RNAi) should be considered with care.

The increased lifespan of odr-3; ife-2 might be simply explained by the combined effect of two genes acting in distinct pathways. However, since the daf-16 mutation is epistatic to the $o d r-3$ mutation and greatly reduces the lifespan of ife-2 knock-down animals, a more complex interaction is also possible. ODR-3 is expressed in 5 pairs of sensory neurons. In the AWA and AWC neurons, ODR-3 functions in perception and transduction of odor signals [37], in ADF and ASH mediates gustatory plasticity and detection of nociceptive stimuli, respectively [37,68], whereas in AWC neurons is involved in temperature sensing [69]. Among these neurons, only AWA and AWC neurons were found to modulate lifespan [36], therefore it is thought that ODR-3 regulates longevity by functioning in these neurons. However, ODR-3 has a role in modulating the adaptive behavior to different stimuli by adjusting the levels of second messenger cGMP in response to environmental cues. We found that WT C. elegans grown on OP50 and HT115 diets have similar lifespans (20.6 mean lifespan on HT115 vs. 19.9 mean lifespan on OP50), indicating an efficient adaptive response of WT animals to these bacterial diets, as previously reported [70, 71]. In contrast, odr-3(n1605) mutants have an increased lifespan on HT115 diet (26\% increase in mean lifespan on HT115 diet, comparative to $7.5 \%$ increase of mean lifespan on OP50 diet), which implicates ODR-3 in metabolic adaptation as it was found in the case of other metabolic genes [70, 71]. IFE-2 is expressed in all soma cells, including neurons [42]. The most pronounced lifespan and healthspan extension of $o d r-3$; ife-2 animals was observed when ife- 2 was down regulated by RNAi. Since RNAi interference is known to affect all tissues of the WT animals with the exception of neurons, these findings raise the possibility that non-neuronal silencing of ife2 by RNAi might be primarily responsible for the improved healthspan and extended longevity of the odr-3; ife-2(RNAi) animals. Alternatively, impaired protein synthesis in neurons due to ife-2 deficiency might be detrimental to nematode health by affecting neuronal proteostasis [72]. Growing evidence unravels an important role for cell non-autonomous regulation of proteostasis in aging in which neuronal activation of stress response pathways such as heat shock response, mitochondrial and ER unfolded protein responses regulate nematode longevity by modulating cellular proteostasis in distal cells $[73,74]$.

DAF-16 stabilizes the transcriptome against the proteostasis collapse during aging by controlling the activity of hundreds of genes, integrating inputs from the DAF-2 pathway and from pathways that appear to regulate lifespan independently of DAF-2 [34, 75]. Therefore, the genetic interaction between odr-3, ife-2 and daf-16 could take many forms. In our experiments, although ife-2 inactivation increased the lifespan of daf16(mu86) mutants, a result that is in accordance with a previous report [42], it did not extend the lifespan beyond that of WT controls. We found that in contrast to $o d r-3$ mutation, which weakly activates DAF-16 in posterior intestine, down regulation of ife-2 does not induce DAF-16 nuclear translocalization, implying that DAF-16 activity is not directly modulated by IFE-2. Both DAF-16 and IFE-2 could affect common processes such as metabolic remodeling and maintenance of cellular proteostasis that modulate longevity. Several metabolic changes were identified as fingerprints for long-lived mutants including the shift from carbon to amino acid catabolism as an alternative energy source, upregulation of lipid storage, increased purine metabolism and increased trehalose stores [43, 76, 77]. Many of these processes were found to be regulated in a DAF-16-dependent manner [75, 78-81]. In mev- 1 mutants, which lack succinate dehydrogenase cytochrome $\mathrm{b}$, depletion of ife-2 induces stress resistance but also restores WT lifespan [42]. A metabolomic study revealed that ife-2 deficiency does not revert the mitochondrial mev-1 defects, but rather restores the catabolism of purine nucleotides (e.g. GMP and AMP) and the metabolism of very long-chain fatty acids (VLFA) [82], processes related to peroxisomes. Since beta-oxidation of VLFA is a source of reactive oxygen species, and peroxisomes are sensitive to increased oxidative stress, ife-2 depletion could also protect peroxisomes from oxidative stress, hence ameliorating peroxisomal function.

We found that the odr-3; ife-2 double mutants are less sensitive to induced $\mathrm{ROS}$ or $\mathrm{H}_{2} \mathrm{O}_{2}$, however a relationship between this and the additive/synergistic nature of the combined intervention cannot be directly 
inferred. First, it was previously shown that stress resistance and lifespan can be experimentally dissociated and the magnitudes of changes in these two parameters produced by mutations are not identical [83]. Second, while it might be intuitive to suggest that the lack of additivity in the oxidative stress defence could mean the two genetic interventions activate the same mechanism, this is highly speculative, and small added differences in stress resistance could in fact affect longevity non linearly.

We also found that the decrease of motility and pharyngeal pumping, which decline in an age-related manner, were delayed in the odr-3; ife-2 mutants. As seen in Figure 4E, the time spent by odr-3; ife-2 animals (in absolute values) in a frail state does not increase, although their lifespan increases compared to both WT and single mutants. Together with the fact that the double intervention extends both mean and maximum lifespan (Supplementary Table 1), it suggests that animals remain healthy for a longer period, while the physiological decline that occurs during the advanced stage of aging is seemingly unaffected. Using an analogy to the socio-economic implications in a human population (if such an intervention could be translatable), such a therapy would probably not reduce the healthcare costs during late senescence, however it would increase the Healthy Life Years (HLY) indicator, which is a measure of productivity during life and an important economic factor.

Among the three genes that we investigated, the role of ife-2 in aging was the most comprehensively analyzed, so far. Thus, it was shown that the long-lived mutants, daf-2, age-1, let-363, clk-1, eat-2, dramatically extended the lifespan of ife-2 impaired animals [21, 42]. There is limited information about interaction of $o d r-3$ or $c k u-70$ with other long-lived mutants. Both, odr3(n1605) and cku-70RNAi extended the lifespan of daf2(e1370) mutants $[39,46]$. We found that mutation in $o d r-3$ extended the healthspan of ife-2 downregulated animals with a higher magnitude than it extended ife-2 lifespan, suggesting that the effect of odr-3 and ife-2 impairment may not be due to a role of these genes in the control of longevity per se, but rather a consequence of a longer healthspan due to amelioration of agerelated decline of physiological processes. This is supported by the observation that in contrast to eat-2 mutants which have reduced pharyngeal pumping, the odr-3; ife-2 animals exhibit a delay in the pharyngeal pumping decline, in older animals.

While much more work is probably needed to fully explore the mechanistic way in which the interaction between odr-3 and ife-2 modulates longevity, our results show that the knock-down of both odr-3 and ife-
2 increases resistance to some types of stress and additively extends lifespan and healthspan.

\section{MATERIALS AND METHODS}

\section{Strains and culture conditions}

The following strains used in this study were provided by the Caenorhabditis Genetic Center (CGC): C. elegans wild-type Bristol strain (N2), CX3222 odr-3(n1605)V, RB579 ife-2(ok306), CF1038 daf-16(mu86)I, OH16024 daf-16(ot971 [daf-16::GFP])I, E. coli OP50 and HT115(DE3) strains. The C. elegans strains were maintained at $20^{\circ} \mathrm{C}$ using standard methods [84].

Multiple mutants were obtained by standard genetic methods and the presence of mutations was tested either by screening for characteristic phenotypes or via PCR genotyping. The homozygous odr-3(n1605) allele was confirmed by negative chemotaxis tests to isoamyl alcohol. To confirm the presence of homozygous daf16(mu86) allele, high density populations were allowed dauer formation and subsequently tested for resistance to SDS $1 \%$. Presence of ife-2(ok306) deletion was confirmed by PCR genotyping.

\section{ife-2 and $c k u-70$ RNAi}

For the RNAi-mediated gene knock-down by feeding method, a slightly modified protocol of the Ahringer technique was used [85]. Briefly, bacteria were grown overnight in LB medium supplemented with $50 \mu \mathrm{g} / \mathrm{ml}$ ampicillin and seeded onto NGM plates supplemented with $25 \mu \mathrm{g} / \mathrm{ml}$ carbenicillin and $1 \mathrm{mM}$ IPTG. The plates were kept at room temperature for two days before use. Several L4 hermaphrodites picked from plates seeded with OP50 were placed onto RNAi plates, transferred the next day to other fresh RNAi plates, allowed to lay eggs for $24 \mathrm{~h}$, then removed. The L4 hermaphrodites developed from eggs laid onto RNAi plates were used for longevity and healthspan assays. For double RNAi experiments, the plates were prepared in a similar way, with the exception that the plates were seeded with a 1:1 mixture of both RNAi bacterial clones. The ife-2 and cku-70 RNAi clones were obtained from the Ahringer RNAi library (Source BioScience, Nottingham, UK); both clones were validated by sequencing. The HT115 bacteria transformed with the L4440 empty vector, HT115 (EV), was used as control for RNAi experiments unless otherwise specified. When ife-2; $c k u-70$ double RNAi was employed, the control worms were grown on plates seeded with a 1:1 density mixture of HT115 (EV) bacteria and ife-2 or cku-70 RNAi clone, respectively, to maintain the same concentration of each double strand RNA as in the strains subjected to double RNAi. 


\section{Lifespan assays}

Since the age at which a treatment is started can significantly influence the outcome [86], all worm cohorts used in this work have been age-synchronized (L4 larvae stage). For all RNAi experiments, agesynchronized L4 larvae were manually transferred to RNAi agar plates containing $15 \mu \mathrm{M}$ 5-fluorodeoxyuridine (FUdR). For lifespan assays of mutant animals, age-synchronized L4 larvae were manually transferred to NGM plates, and seeded with OP50. In case of lifespan assays without FUdR, the worms were transferred to a new plate every day until they ceased laying eggs, then when needed. For mutant animals cultured with FUdR, a $15 \mu \mathrm{M}$ FUdR concentration (same as in the RNAi experiments) was used. In all cases, worms were kept at $20^{\circ} \mathrm{C}$ and scored daily as dead or alive based on their response to a gentle touch with a wire. Worms that presented externalization of internal organs, died because of bagging, or crawled up the wall of the dish were censored. For RNAi experiments, the WT control and odr-3 animals were fed with HT115 (EV) bacteria. 85 worms were assayed per experiment.

While the lifespan assays were not conducted in a blinded manner, as suggested by Gruber et al., [87], the experiments were carried out by 3 operators, working with the data independently and results were evaluated for consistency. From the beginning of the study, all operators aimed to treat worm cohorts in an unbiased fashion and keep them in the same conditions.

\section{Locomotion assay}

Animals were scored for free movement and for a response to prodding with a wire, daily, during the lifespan assay until death. Worms were classified into three motion stages, based on ability to engage and coordinate the body wall muscle in a forward or backward movement according to a previously described method [88], with slight modifications. The three stages considered were: 1) state A, corresponding to a physiological, fully mobile state, which included animals that could move more than $0.5 \mathrm{~cm}$ (freely or upon prodding); 2) state $\mathrm{B}$, representing impaired animals that responded to the prodding, but did not have enough strength to move more than $0.5 \mathrm{~cm}$, and 3) state $\mathrm{C}$, which encompassed animals in a frailty state that barely exhibited head or tail movements or twitches upon prodding.

\section{Pharyngeal pumping assay}

For the pharyngeal pumping assay, separate worm cohorts were cultured, in three independent experiments, each with 60 animals grown on FUdRsupplemented plates seeded with OP50 bacteria. Pumping was monitored and recorded at 1, 5, 10 and 15 days post-L4 moult by filming 13-15 randomly selected worms at each time point. Time lapse recordings were obtained on the Zeiss SteREO Discovery. V20 stereomicroscope (Carl Zeiss AG, Jena, Germany) using the AxioVs40 V4.8.2.0 software (Carl Zeiss AG), using the $1 \mathrm{X}$ Plan Apo S objective, at a magnification of 150X; the time lapse captures were converted into.mp4 files using an in-house developed script. Pharyngeal contractions were then accurately counted during a 30seconds interval. For each strain the average number of pharyngeal movements per minute, standard deviation and standard error of the mean were computed.

\section{Stress assays and fecundity}

Tolerance to heat and oxidative stress was tested for late L4 animals, since responses to both stresses become repressed early in adulthood (starting as early as 4 hours post L4) [89], suggesting that collapse of cellular stress response could represent an early molecular event in the aging process.

\section{Heat stress assay}

To obtain synchronized populations, five hermaphrodites were let to lay eggs for about two hours on three replicate plates. The larvae were reared at $20^{\circ} \mathrm{C}$ up to late L4 stage, shifted to $35^{\circ} \mathrm{C}$ for four hours, then returned to $20^{\circ} \mathrm{C}$. The percentage of alive animals was scored $48 \mathrm{~h}$ later. More than 420 animals were tested for each strain in three individual experiments.

\section{Oxidative stress assay}

Tolerance to oxidative stress was tested by exposure to paraquat and $\mathrm{NaN}_{3}$. Both assays were essentially performed as previously described [42]. Briefly, synchronized L4 larvae were transferred to NGM plates containing $2 \mathrm{mM}$ paraquat and survival was scored on day 5 of exposure. For $\mathrm{NaN}_{3}$ tolerance, synchronized L4 larvae were collected, washed with M9, incubated for one hour with $0.5 \mathrm{M}$ freshly made $\mathrm{NaN}_{3}$ in $\mathrm{M} 9$, washed with M9 and placed on a NGM plate to recover. Survival rates were determined after 24 hours.

\section{Brood size}

To analyze the total brood size, L4 worms were placed on NGM plates seeded with OP50 and transferred every $12 \mathrm{~h}$ to a new plate until they ceased laying eggs. The number of eggs laid by each worm was counted after removal of the parent.

\section{DAF-16::GFP nuclear translocation}

To verify DAF-16 activation we used the CRISPR allele of daf-16 tagged at the C-terminus with GFP [90]. Since 
DAF-16 translocation from cytoplasm to nucleus is induced by heat stress and common drugs used to anesthetize the worms [91], the young adult worms were fixed in $4 \%$ paraformaldehyde. The worms were grown at $20 \mathrm{o} \mathrm{C}$ on RNAi plates, at L4 stage $15 \mu \mathrm{M}$ FUdR was added and next day, the young adults were fixed and immediately visualized. Images were acquired with a Zeiss LSM 710 laser scanning confocal microscope using 10x objective, Argon 488 laser, and identical acquisition setting.

\section{Statistical analysis}

Comparisons between the lifespan values of different strains were carried out by analyzing Kaplan-Meier survival curves. For the statistical analysis and graphical representation of the curves, the R package "survival" was used (https://cran.r-project.org/package=survival).

For the statistical analysis of locomotion, animals were scored by motion stage and plotted as motility curves, similar to the lifespan curves, to evaluate the decline rate of motility in each strain. While in the survival analysis an event represents the death of one worm, in the locomotion analysis an event was defined as the transition between motility states, modeling the population dynamics from increased to low motility (Supplementary Figure 4). Since three motility categories exist, two different motility analyses were conducted, corresponding to two types of events: i) transitions from the mobile state $A$ to either the impaired state $\mathrm{B}$ or to the frail state $\mathrm{C}$, and ii) transitions from either state A or B to state C. In the manuscript, only the results from the motility analysis of transitions from $\mathrm{A}$ vs. cumulated $\mathrm{B}$ and $\mathrm{C}$ is included, however the two analyses produced very similar results (data not shown).

For all survival and locomotion analyses, the statistical significance was tested using the log-rank test (MantelCox). Comparisons were performed against WT, WT(EV) or daf-16, as appropriately. If not otherwise specified, $\mathrm{p}<0.0001$ was considered significant. The $\mathrm{p}-$ values were corrected for multiple testing using the Benjamini-Hochberg method, at alpha $=0.05$.

One-way ANOVA was used for the analysis of pharyngeal pumping, heat, paraquat and sodium azide stress resistance assays. All mutants were compared to wild type and statistical significance was assessed using Dunnett's test.

\section{AUTHOR CONTRIBUTIONS}

Conceptualization, RT and SG; methodology, SG; lab work and validations, IVM, VNCS, SG; bioinformatics and programmatic tools, GB; all authors have participated to the analysis of results and to the writing of the manuscript; supervision, SG and RT; funding acquisition, RT. All authors have read and agreed to the published version of the manuscript.

\section{ACKNOWLEDGMENTS}

Some of the strains used in this study were provided by the CGC, which is funded by NIH Office of Research Infrastructure Programs (P40 OD010440). The authors would like to thank Anton Kulaga for helping with the development of the time-lapse conversion script.

\section{CONFLICTS OF INTEREST}

The authors declare that they have no conflicts of interest.

\section{FUNDING}

This work was supported by the National Authority for Scientific Research and Innovation, and by the Ministry of European Funds, Romania, through the Competitiveness Operational Programme 2014-2020, POC-A.1-A.1.1.4-E-2015 [Grant number: 40/02.09.2016, ID: P_37_778, to RT].

\section{REFERENCES}

1. Phillip JM, Aifuwa I, Walston J, Wirtz D. The Mechanobiology of Aging. Annu Rev Biomed Eng. 2015; 17:113-41. https://doi.org/10.1146/annurev-bioeng-071114040829 PMID:26643020

2. Lemoine M. Defining aging. Biol Philos. 2020; 35:46. https://doi.org/10.1007/s10539-020-09765-z

3. Butler RN, Miller RA, Perry D, Carnes BA, Williams TF, Cassel C, Brody J, Bernard MA, Partridge L, Kirkwood T, Martin GM, Olshansky SJ. New model of health promotion and disease prevention for the 21st century. BMJ. 2008; 337:a399.

https://doi.org/10.1136/bmj.a399 PMID:18614506

4. Miller RA. "Dividends" from research on aging--can biogerontologists, at long last, find something useful to do? J Gerontol A Biol Sci Med Sci. 2009; 64:157-60. https://doi.org/10.1093/gerona/gln062 PMID:19225032

5. High KP. Infrastructure and resources for an aging population: embracing complexity in translational research. Transl Res. 2014; 163:446-55. https://doi.org/10.1016/j.trsl.2013.09.001 PMID:24095640

6. Kenyon CJ. The genetics of ageing. Nature. 2010; 464:504-12.

https://doi.org/10.1038/nature08980

PMID:20336132 
7. Partridge L, Deelen J, Slagboom PE. Facing up to the global challenges of ageing. Nature. 2018; 561:45-56. https://doi.org/10.1038/s41586-018-0457-8 PMID:30185958

8. Lezzerini M, Smith RL, Budovskaya Y. Developmental drift as a mechanism for aging: lessons from nematodes. Biogerontology. 2013; 14:693-701. https://doi.org/10.1007/s10522-013-9462-3 PMID:24122213

9. Tacutu R, Thornton D, Johnson E, Budovsky A, Barardo D, Craig T, Diana E, Lehmann G, Toren D, Wang J, Fraifeld VE, de Magalhães JP. Human Ageing Genomic Resources: new and updated databases. Nucleic Acids Res. 2018; 46:D1083-90. https://doi.org/10.1093/nar/gkx1042 PMID:29121237

10. Yanai H, Budovsky A, Barzilay T, Tacutu R, Fraifeld VE. Wide-scale comparative analysis of longevity genes and interventions. Aging Cell. 2017; 16:1267-75. https://doi.org/10.1111/acel.12659 PMID:28836369

11. Toren D, Kulaga A, Jethva M, Rubin E, Snezhkina AV, Kudryavtseva AV, Nowicki D, Tacutu R, Moskalev AA, Fraifeld VE. Gray whale transcriptome reveals longevity adaptations associated with DNA repair and ubiquitination. Aging Cell. 2020; 19:e13158. https://doi.org/10.1111/acel.13158 PMID:32515539

12. Kulaga AY, Ursu E, Toren D, Tyshchenko V, Guinea R, Pushkova M, Fraifeld VE, Tacutu R. Machine Learning Analysis of Longevity-Associated Gene Expression Landscapes in Mammals. Int J Mol Sci. 2021; 22:1073. https://doi.org/10.3390/ijms22031073 PMID:33499037

13. Kenyon C, Chang J, Gensch E, Rudner A, Tabtiang R. A C. elegans mutant that lives twice as long as wild type. Nature. 1993; 366:461-64.

https://doi.org/10.1038/366461a0 PMID:27153

14. Fabrizio P, Gattazzo C, Battistella L, Wei M, Cheng C, McGrew K, Longo VD. Sir2 blocks extreme life-span extension. Cell. 2005; 123:655-67.

https://doi.org/10.1016/i.cell.2005.08.042 PMID:16286010

15. Ayyadevara S, Alla R, Thaden JJ, Shmookler Reis RJ. Remarkable longevity and stress resistance of nematode PI3K-null mutants. Aging Cell. 2008; 7:13-22.

https://doi.org/10.1111/j.1474-9726.2007.00348.x

PMID:17996009

16. Phillips PC. Epistasis--the essential role of gene interactions in the structure and evolution of genetic systems. Nat Rev Genet. 2008; 9:855-67.

https://doi.org/10.1038/nrg2452

PMID:18852697
17. Mackay TF. Epistasis and quantitative traits: using model organisms to study gene-gene interactions. Nat Rev Genet. 2014; 15:22-33. https://doi.org/10.1038/nrg3627 PMID:24296533

18. Bunu G, Toren D, Ion CF, Barardo D, Sârghie L, Grigore LG, de Magalhães JP, Fraifeld VE, Tacutu R. SynergyAge, a curated database for synergistic and antagonistic interactions of longevity-associated genes. Sci Data. 2020; 7:366.

https://doi.org/10.1038/s41597-020-00710-z PMID:33106474

19. Martins R, Lithgow GJ, Link W. Long live FOXO: unraveling the role of FOXO proteins in aging and longevity. Aging Cell. 2016; 15:196-207.

https://doi.org/10.1111/acel.12427

PMID:26643314

20. Bitto A, Wang AM, Bennett CF, Kaeberlein M. Biochemical Genetic Pathways that Modulate Aging in Multiple Species. Cold Spring Harb Perspect Med. 2015; 5:a025114.

https://doi.org/10.1101/cshperspect.a025114 PMID:26525455

21. Hansen M, Taubert S, Crawford D, Libina N, Lee SJ, Kenyon C. Lifespan extension by conditions that inhibit translation in Caenorhabditis elegans. Aging Cell. 2007; 6:95-110. https://doi.org/10.1111/j.1474-9726.2006.00267.x PMID:17266679

22. Van Raamsdonk JM, Hekimi S. Deletion of the mitochondrial superoxide dismutase sod-2 extends lifespan in Caenorhabditis elegans. PLoS Genet. 2009; 5:e1000361.

https://doi.org/10.1371/journal.pgen.1000361 PMID:19197346

23. Lakowski B, Hekimi S. Determination of life-span in Caenorhabditis elegans by four clock genes. Science. 1996; 272:1010-13.

https://doi.org/10.1126/science.272.5264.1010 PMID: 8638122

24. Chen D, Li PW, Goldstein BA, Cai W, Thomas EL, Chen F, Hubbard AE, Melov S, Kapahi P. Germline signaling mediates the synergistically prolonged longevity produced by double mutations in daf-2 and rsks-1 in C. elegans. Cell Rep. 2013; 5:1600-10. https://doi.org/10.1016/i.celrep.2013.11.018 PMID:24332851

25. Yang CC, Chen D, Lee SS, Walter L. The dynaminrelated protein DRP-1 and the insulin signaling pathway cooperate to modulate Caenorhabditis elegans longevity. Aging Cell. 2011; 10:724-28. https://doi.org/10.1111/j.1474-9726.2011.00711.x PMID:21463460 
26. Van Raamsdonk JM, Meng Y, Camp D, Yang W, Jia X, Bénard C, Hekimi S. Decreased energy metabolism extends life span in Caenorhabditis elegans without reducing oxidative damage. Genetics. 2010; 185:559-71.

https://doi.org/10.1534/genetics.110.115378

PMID:20382831

27. Apfeld J, Kenyon C. Regulation of lifespan by sensory perception in Caenorhabditis elegans. Nature. 1999; 402:804-09.

https://doi.org/10.1038/45544 PMID:10617200

28. Gottlieb S, Ruvkun G. daf-2, daf-16 and daf-23: genetically interacting genes controlling Dauer formation in Caenorhabditis elegans. Genetics. 1994; 137:107-20.

https://doi.org/10.1093/genetics/137.1.107 PMID:이요

29. Gems D, Sutton AJ, Sundermeyer ML, Albert PS, King $\mathrm{KV}$, Edgley ML, Larsen PL, Riddle DL. Two pleiotropic classes of daf-2 mutation affect larval arrest, adult behavior, reproduction and longevity in Caenorhabditis elegans. Genetics. 1998; 150:129-55.

https://doi.org/10.1093/genetics/150.1.129

PMID: $\underline{9725835}$

30. Kimura KD, Tissenbaum HA, Liu Y, Ruvkun G. daf-2, an insulin receptor-like gene that regulates longevity and diapause in Caenorhabditis elegans. Science. 1997; 277:942-46.

https://doi.org/10.1126/science.277.5328.942 PMID:

31. Kimura KD, Riddle DL, Ruvkun G. The C. elegans DAF-2 insulin-like receptor is abundantly expressed in the nervous system and regulated by nutritional status. Cold Spring Harb Symp Quant Biol. 2011; 76:113-20. https://doi.org/10.1101/sqb.2011.76.010660 PMID:22123849

32. Lin K, Dorman JB, Rodan A, Kenyon C. daf-16: An HNF$3 /$ forkhead family member that can function to double the life-span of Caenorhabditis elegans. Science. 1997; 278:1319-22.

https://doi.org/10.1126/science.278.5341.1319 PMID: $\underline{9360933}$

33. Ogg S, Paradis S, Gottlieb S, Patterson GI, Lee L, Tissenbaum HA, Ruvkun G. The Fork head transcription factor DAF-16 transduces insulin-like metabolic and longevity signals in C. elegans. Nature. 1997; 389:994-99. https://doi.org/10.1038/40194 PMID:9353126

34. Tissenbaum HA. DAF-16: FOXO in the Context of C. elegans. Curr Top Dev Biol. 2018; 127:1-21. https://doi.org/10.1016/bs.ctdb.2017.11.007 PMID:29433733
35. Bargmann $\mathrm{Cl}$, Hartwieg E, Horvitz HR. Odorantselective genes and neurons mediate olfaction in C. elegans. Cell. 1993; 74:515-27.

https://doi.org/10.1016/0092-8674(93)80053-h

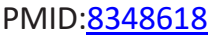

36. Alcedo J, Kenyon C. Regulation of C. elegans longevity by specific gustatory and olfactory neurons. Neuron. 2004; 41:45-55.

https://doi.org/10.1016/s0896-6273(03)00816-x PMID:14715134

37. Roayaie K, Crump JG, Sagasti A, Bargmann Cl. The G alpha protein ODR-3 mediates olfactory and nociceptive function and controls cilium morphogenesis in C. elegans olfactory neurons. Neuron. 1998; 20:55-67. https://doi.org/10.1016/s0896-6273(00)80434-1 PMID:9459442

38. Lans H, Rademakers S, Jansen G. A network of stimulatory and inhibitory Galpha-subunits regulates olfaction in Caenorhabditis elegans. Genetics. 2004; 167:1677-87.

https://doi.org/10.1534/genetics.103.024786 PMID:15342507

39. Lans $\mathrm{H}$, Jansen $\mathrm{G}$. Multiple sensory $\mathrm{G}$ proteins in the olfactory, gustatory and nociceptive neurons modulate longevity in Caenorhabditis elegans. Dev Biol. 2007; 303:474-82.

https://doi.org/10.1016/i.ydbio.2006.11.028 PMID: 17187771

40. Mak HY, Nelson LS, Basson M, Johnson CD, Ruvkun G. Polygenic control of Caenorhabditis elegans fat storage. Nat Genet. 2006; 38:363-68.

https://doi.org/10.1038/ng1739

PMID:16462744

41. Shields HJ, Traa A, Van Raamsdonk JM. Beneficial and Detrimental Effects of Reactive Oxygen Species on Lifespan: A Comprehensive Review of Comparative and Experimental Studies. Front Cell Dev Biol. 2021; 9:628157. https://doi.org/10.3389/fcell.2021.628157 PMID:33644065

42. Syntichaki P, Troulinaki K, Tavernarakis N. elF4E function in somatic cells modulates ageing in Caenorhabditis elegans. Nature. 2007; 445:922-26. https://doi.org/10.1038/nature05603 PMID:17277769

43. Fuchs S, Bundy JG, Davies SK, Viney JM, Swire JS, Leroi AM. A metabolic signature of long life in Caenorhabditis elegans. BMC Biol. 2010; 8:14.

https://doi.org/10.1186/1741-7007-8-14 PMID:20146810

44. Chen F, Peterson SR, Story MD, Chen DJ. Disruption of DNA-PK in Ku80 mutant $x r s-6$ and the implications in 
DNA double-strand break repair. Mutat Res. 1996; 362:9-19.

https://doi.org/10.1016/0921-8777(95)00026-7 PMID: $\underline{8538653}$

45. Clejan I, Boerckel J, Ahmed S. Developmental modulation of nonhomologous end joining in Caenorhabditis elegans. Genetics. 2006; 173:1301-17. https://doi.org/10.1534/genetics.106.058628 PMID:16702421

46. McColl G, Vantipalli MC, Lithgow GJ. The C. elegans ortholog of mammalian Ku70, interacts with insulinlike signaling to modulate stress resistance and life span. FASEB J. 2005; 19:1716-18. https://doi.org/10.1096/fj.04-2447fje PMID:16099946

47. Sun $X$, Chen WD, Wang YD. DAF-16/FOXO Transcription Factor in Aging and Longevity. Front Pharmacol. 2017; 8:548.

https://doi.org/10.3389/fphar.2017.00548 PMID:28878670

48. Chen AT, Guo C, Itani OA, Budaitis BG, Williams TW, Hopkins CE, McEachin RC, Pande M, Grant AR, Yoshina S, Mitani S, Hu PJ. Longevity Genes Revealed by Integrative Analysis of Isoform-Specific daf-16/FoxO Mutants of Caenorhabditis elegans. Genetics. 2015; 201:613-29.

https://doi.org/10.1534/genetics.115.177998

PMID:26219299

49. Aitlhadj L, Stürzenbaum SR. The use of FUdR can cause prolonged longevity in mutant nematodes. Mech Ageing Dev. 2010; 131:364-65.

https://doi.org/10.1016/j.mad.2010.03.002

PMID:20236608

50. Van Raamsdonk JM, Hekimi S. FUdR causes a twofold increase in the lifespan of the mitochondrial mutant gas-1. Mech Ageing Dev. 2011; 132:519-21. https://doi.org/10.1016/j.mad.2011.08.006 PMID:21893079

51. Davies SK, Leroi AM, Bundy JG. Fluorodeoxyuridine affects the identification of metabolic responses to daf2 status in Caenorhabditis elegans. Mech Ageing Dev. 2012; 133:46-49.

https://doi.org/10.1016/i.mad.2011.11.002

PMID:22116032

52. Huang C, Xiong C, Kornfeld K. Measurements of agerelated changes of physiological processes that predict lifespan of Caenorhabditis elegans. Proc Natl Acad Sci USA. 2004; 101:8084-89.

https://doi.org/10.1073/pnas.0400848101 PMID:15141086

53. Dues DJ, Andrews EK, Schaar CE, Bergsma AL, Senchuk MM, Van Raamsdonk JM. Aging causes decreased resistance to multiple stresses and a failure to activate specific stress response pathways. Aging (Albany NY). 2016; 8:777-95. https://doi.org/10.18632/aging.100939 PMID:27053445

54. López-Otín C, Kroemer G. Hallmarks of Health. Cell. 2021; 184:33-63. https://doi.org/10.1016/i.cell.2020.11.034 PMID:33340459

55. Gruber J, Tang SY, Halliwell B. Evidence for a trade-off between survival and fitness caused by resveratrol treatment of Caenorhabditis elegans. Ann N Y Acad Sci. 2007; 1100:530-42. https://doi.org/10.1196/annals.1395.059 PMID:17460219

56. Aguilaniu H. The mysterious relationship between reproduction and longevity. Worm. 2015; 4:e1020276. https://doi.org/10.1080/21624054.2015.1020276 PMID:26430561

57. Mukhopadhyay A, Tissenbaum HA. Reproduction and longevity: secrets revealed by $C$. elegans. Trends Cell Biol. 2007; 17:65-71.

https://doi.org/10.1016/j.tcb.2006.12.004 PMID: 17187981

58. López-Otín C, Blasco MA, Partridge L, Serrano M, Kroemer G. The hallmarks of aging. Cell. 2013; 153:1194-217.

https://doi.org/10.1016/i.cell.2013.05.039 PMID:23746838

59. Möller S, Saul N, Cohen AA, Köhling R, Sender S, Murua Escobar $\mathrm{H}$, Junghanss $\mathrm{C}$, Cirulli F, Berry A, Antal P, Adler $P$, Vilo J, Boiani $M$, et al. Healthspan pathway maps in C. elegans and humans highlight transcription, proliferation/biosynthesis and lipids. Aging (Albany NY). 2020; 12:12534-81.

https://doi.org/10.18632/aging.103514 PMID:32634117

60. Tacutu R, Shore DE, Budovsky A, de Magalhães JP, Ruvkun G, Fraifeld VE, Curran SP. Prediction of C. elegans longevity genes by human and worm longevity networks. PLoS One. 2012; 7:e48282.

https://doi.org/10.1371/journal.pone.0048282 PMID:23144747

61. Calvert S, Tacutu R, Sharifi S, Teixeira R, Ghosh P, de Magalhães JP. A network pharmacology approach reveals new candidate caloric restriction mimetics in C. elegans. Aging Cell. 2016; 15:256-66. https://doi.org/10.1111/acel.12432 PMID:26676933

62. Wolfson M, Budovsky A, Tacutu R, Fraifeld V. The signaling hubs at the crossroad of longevity and agerelated disease networks. Int J Biochem Cell Biol. 2009; 41:516-20. 
https://doi.org/10.1016/i.biocel.2008.08.026 PMID:18793745

63. Tacutu R, Budovsky A, Fraifeld VE. The NetAge database: a compendium of networks for longevity, age-related diseases and associated processes. Biogerontology. 2010; 11:513-22.

https://doi.org/10.1007/s10522-010-9265-8

PMID:20186480

64. Tacutu R, Budovsky A, Wolfson M, Fraifeld VE. MicroRNA-regulated protein-protein interaction networks: how could they help in searching for prolongevity targets? Rejuvenation Res. 2010; 13:373-77. https://doi.org/10.1089/rej.2009.0980 PMID:20367577

65. Admasu TD, Chaithanya Batchu K, Barardo D, Ng LF, Lam VY, Xiao L, Cazenave-Gassiot A, Wenk MR, Tolwinski NS, Gruber J. Drug Synergy Slows Aging and Improves Healthspan through IGF and SREBP Lipid Signaling. Dev Cell. 2018; 47:67-79.e5. https://doi.org/10.1016/i.devcel.2018.09.001 PMID:30269951

66. Abbasi S, Parmar G, Kelly RD, Balasuriya N, SchildPoulter C. The Ku complex: recent advances and emerging roles outside of non-homologous endjoining. Cell Mol Life Sci. 2021; 78:4589-613.

https://doi.org/10.1007/s00018-021-03801-1 PMID:33855626

67. Gong P, Wang Y, Jing Y. Apoptosis Induction byHistone Deacetylase Inhibitors in Cancer Cells: Role of Ku70. Int J Mol Sci. 2019; 20:1601.

https://doi.org/10.3390/ijms20071601

PMID:30935057

68. Hukema RK, Rademakers S, Dekkers MP, Burghoorn J, Jansen G. Antagonistic sensory cues generate gustatory plasticity in Caenorhabditis elegans. EMBO J. 2006; 25:312-22.

https://doi.org/10.1038/sj.emboj.7600940

PMID: 16407969

69. Kuhara A, Okumura M, Kimata T, Tanizawa Y, Takano R, Kimura KD, Inada H, Matsumoto K, Mori I. Temperature sensing by an olfactory neuron in a circuit controlling behavior of C. elegans. Science. 2008; 320:803-07. https://doi.org/10.1126/science.1148922 PMID:18403676

70. Soukas AA, Kane EA, Carr CE, Melo JA, Ruvkun G. Rictor/TORC2 regulates fat metabolism, feeding, growth, and life span in Caenorhabditis elegans. Genes Dev. 2009; 23:496-511. https://doi.org/10.1101/gad.1775409 PMID:19240135

71. Brooks KK, Liang B, Watts JL. The influence of bacterial diet on fat storage in C. elegans. PLoS One. 2009; 4:e7545. https://doi.org/10.1371/journal.pone.0007545 PMID:19844570

72. Klaips CL, Jayaraj GG, Hartl FU. Pathways of cellular proteostasis in aging and disease. J Cell Biol. 2018; 217:51-63. https://doi.org/10.1083/icb.201709072 PMID:29127110

73. Taylor RC, Berendzen KM, Dillin A. Systemic stress signalling: understanding the cell non-autonomous control of proteostasis. Nat Rev Mol Cell Biol. 2014; 15:211-17. https://doi.org/10.1038/nrm3752 PMID:24556842

74. Morimoto RI. Cell-Nonautonomous Regulation of Proteostasis in Aging and Disease. Cold Spring Harb Perspect Biol. 2020; 12:a034074.

https://doi.org/10.1101/cshperspect.a034074 PMID:30962274

75. Li ST, Zhao HQ, Zhang P, Liang CY, Zhang YP, Hsu AL, Dong MQ. DAF-16 stabilizes the aging transcriptome and is activated in mid-aged Caenorhabditis elegans to cope with internal stress. Aging Cell. 2019; 18:e12896.

https://doi.org/10.1111/acel.12896 PMID:30773782

76. Gao AW, Smith RL, van Weeghel M, Kamble R, Janssens GE, Houtkooper RH. Identification of key pathways and metabolic fingerprints of longevity in C. elegans. Exp Gerontol. 2018; 113:128-40.

https://doi.org/10.1016/j.exger.2018.10.003 PMID:30300667

77. Han S, Schroeder EA, Silva-García CG, Hebestreit K, Mair WB, Brunet A. Mono-unsaturated fatty acids link H3K4me3 modifiers to C. elegans lifespan. Nature. 2017; 544:185-90.

https://doi.org/10.1038/nature21686

PMID:28379943

78. Hibshman JD, Doan AE, Moore BT, Kaplan RE, Hung A, Webster AK, Bhatt DP, Chitrakar R, Hirschey MD, Baugh LR. daf-16/FoxO promotes gluconeogenesis and trehalose synthesis during starvation to support survival. Elife. 2017; 6:e30057.

https://doi.org/10.7554/eLife.30057 PMID:29063832

79. Seo Y, Kingsley S, Walker G, Mondoux MA, Tissenbaum HA. Metabolic shift from glycogen to trehalose promotes lifespan and healthspan in Caenorhabditis elegans. Proc Natl Acad Sci USA. 2018; 115:E2791-800. https://doi.org/10.1073/pnas.1714178115 PMID:29511104

80. Amrit FR, Steenkiste EM, Ratnappan R, Chen SW, McClendon TB, Kostka D, Yanowitz J, Olsen CP, Ghazi A. DAF-16 and TCER-1 Facilitate Adaptation to Germline Loss by Restoring Lipid Homeostasis and Repressing 
Reproductive Physiology in C. elegans. PLoS Genet. 2016; 12:e1005788.

https://doi.org/10.1371/journal.pgen.1005788

PMID:26862916

81. Wan QL, Shi X, Liu J, Ding AJ, Pu YZ, Li Z, Wu GS, Luo HR. Metabolomic signature associated with reproduction-regulated aging in Caenorhabditis elegans. Aging (Albany NY). 2017; 9:447-74.

https://doi.org/10.18632/aging.101170

PMID:28177875

82. Jaeger C, Tellström V, Zurek G, König S, Eimer S, Kammerer B. Metabolomic changes in Caenorhabditis elegans lifespan mutants as evident from GC-EI-MS and GC-APCI-TOF-MS profiling. Metabolomics. 2014; 10:859-76. https://doi.org/10.1007/s11306-014-0637-y

83. Dues DJ, Andrews EK, Senchuk MM, Van Raamsdonk JM. Resistance to Stress Can Be Experimentally Dissociated From Longevity. J Gerontol A Biol Sci Med Sci. 2019; 74:1206-14. https://doi.org/10.1093/gerona/gly213 PMID: $\underline{30247515}$

84. Stiernagle T. Maintenance of C. elegans. WormBook. 2006.

https://doi.org/10.1895/wormbook.1.101.1 PMID:18050451

85. Kamath RS, Martinez-Campos M, Zipperlen P, Fraser AG, Ahringer J. Effectiveness of specific RNA-mediated interference through ingested double-stranded RNA in Caenorhabditis elegans. Genome Biol. 2001; 2:RESEARCH0002.

https://doi.org/10.1186/gb-2000-2-1-research0002 PMID:11178279

86. Saul N, Möller S, Cirulli F, Berry A, Luyten W, Fuellen G. Health and longevity studies in C. elegans: the "healthy worm database" reveals strengths, weaknesses and gaps of test compound-based studies. Biogerontology. $2021 ; 22: 215-36$.

https://doi.org/10.1007/s10522-021-09913-2

PMID:33683565

87. Gruber J, Ng LF, Poovathingal SK, Halliwell B. Deceptively simple but simply deceptive-Caenorhabditis elegans lifespan studies: considerations for aging and antioxidant effects. FEBS Lett. 2009; 583:3377-87.

https://doi.org/10.1016/i.febslet.2009.09.051 PMID:19815017

88. Herndon LA, Schmeissner PJ, Dudaronek JM, Brown PA, Listner KM, Sakano Y, Paupard MC, Hall DH, Driscoll $M$. Stochastic and genetic factors influence tissuespecific decline in ageing C. elegans. Nature. 2002; 419:808-14.

https://doi.org/10.1038/nature01135

PMID:12397350

89. Labbadia J, Morimoto RI. Repression of the Heat Shock Response Is a Programmed Event at the Onset of Reproduction. Mol Cell. 2015; 59:639-50. https://doi.org/10.1016/i.molcel.2015.06.027 PMID:26212459

90. Aghayeva U, Bhattacharya A, Hobert O. A panel of fluorophore-tagged daf-16 alleles. MicroPubl Biol. 2020; 2020:10.17912. https://doi.org/10.17912/micropub.biology.000210 PMID:32550509

91. Manjarrez JR, Mailler R. Stress and timing associated with Caenorhabditis elegans immobilization methods. Heliyon. 2020; 6:e04263. https://doi.org/10.1016/i.heliyon.2020.e04263 PMID:32671240 


\section{SUPPLEMENTARY MATERIALS}

\section{Supplementary Figures}

Strains: N2; odr-3(n1605); ife-2(RNAi); cku-70(RNAi)
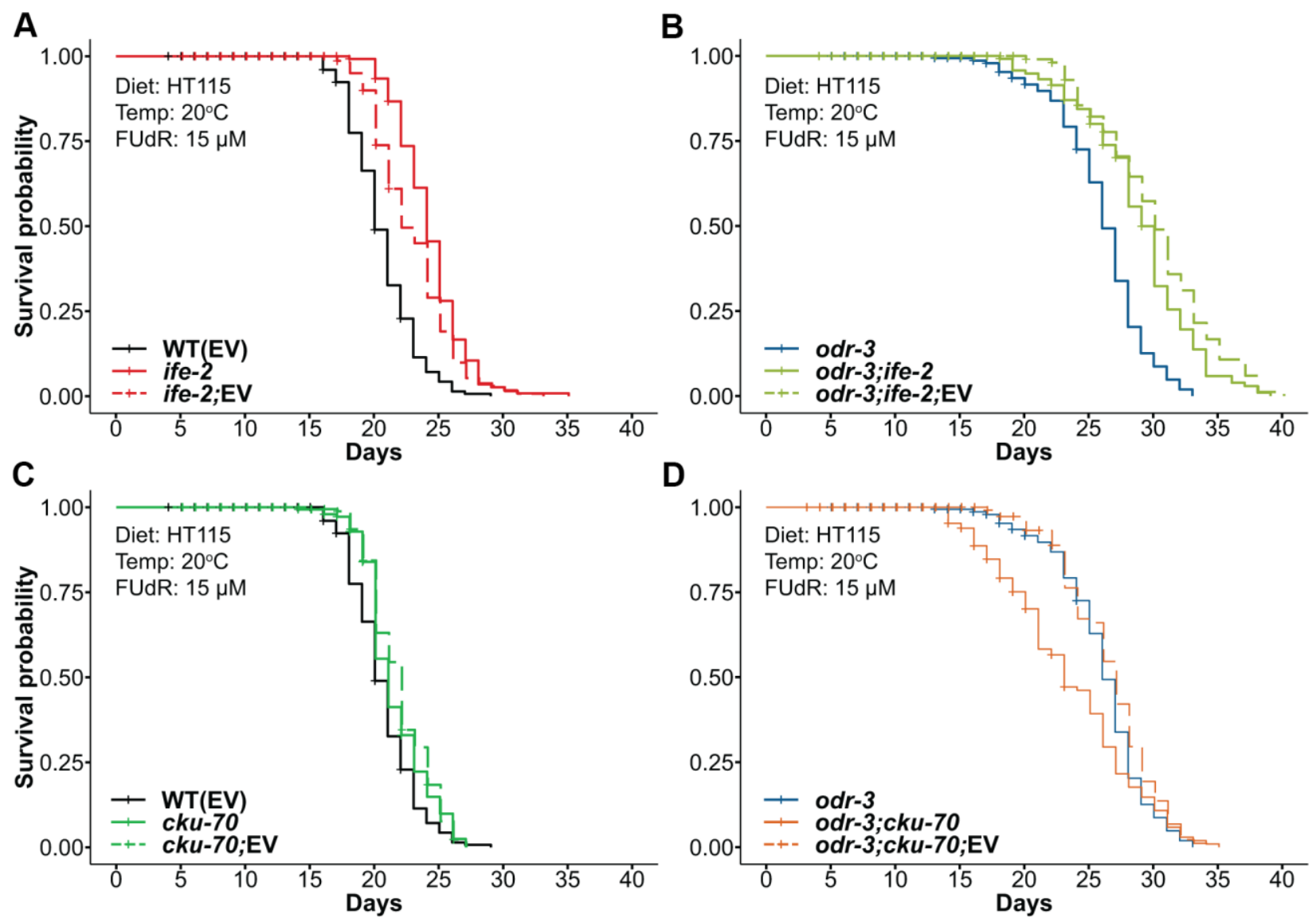

Supplementary Figure 1. Kaplan-Meier survival curves for worms fed bacteria expressing the target dsRNA or an equal mix of target dsRNA and EV. All survival plots represent pooled populations from 3 independent experiments. (A, B) Lifespan comparison of WT (A) or odr-3(n1605) (B) worms fed bacteria expressing ife-2 dsRNA or a 1:1 mixture of ife-2 dsRNA and empty-vector (EV). (C, D) Lifespan comparison of WT (C) or odr-3(n1605) (D) worms fed bacteria expressing $c k u-70$ dsRNA or a mixture of $c k u-70$ dsRNA and EV. Lifespan values are given in Supplementary Table 1. 

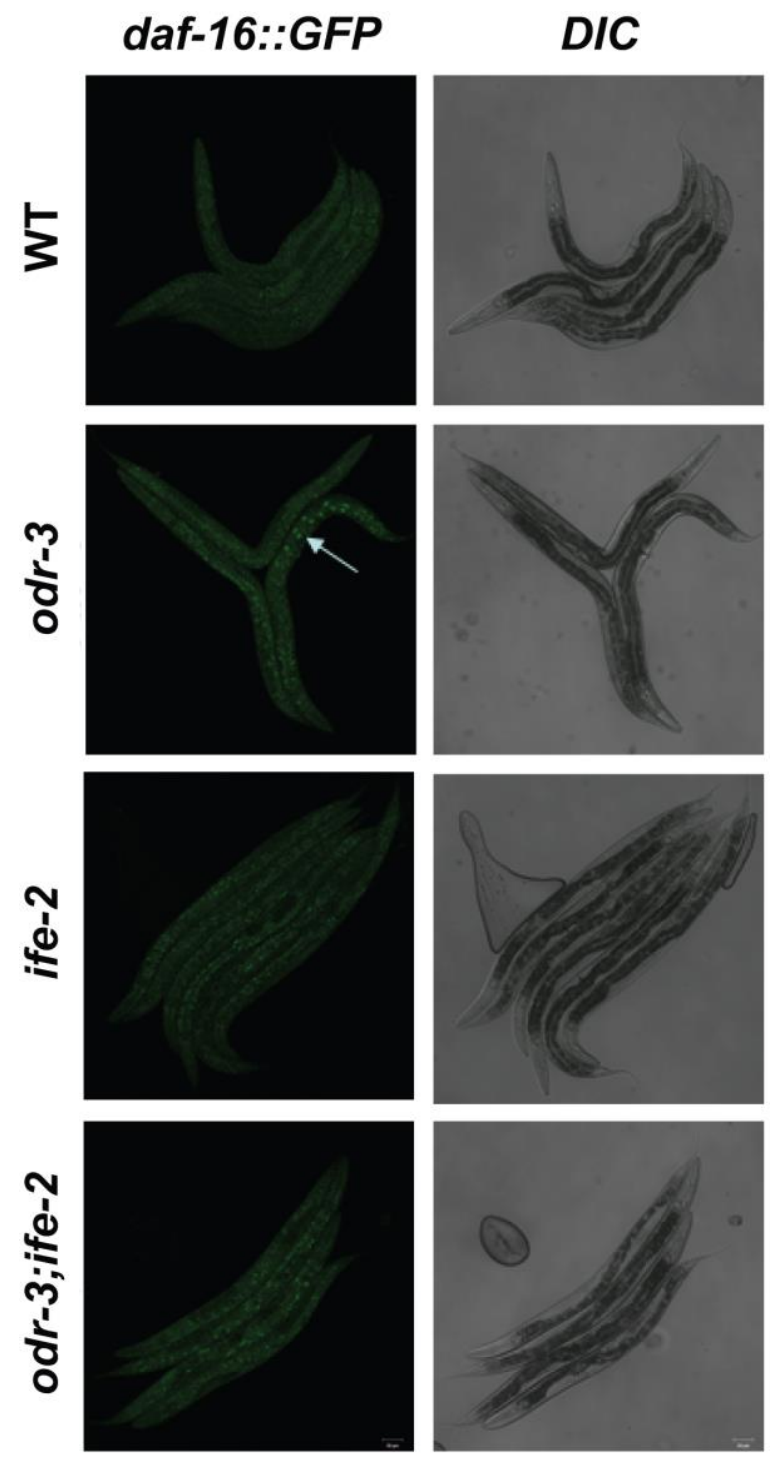

Supplementary Figure 2. DAF-16::GFP nuclear translocation in odr-3; ife-2 (RNAi) mutant worms. Worms expressing daf-16 (ot971 [daf-16::GFP]) fluorescent marker in WT animals and odr-3(n1605), ife-2(RNAi) or odr-3(n1605); ife-2(RNAi) mutant background show nuclear accumulation of DAF-16::GFP in odr-3(n1605) mutants but not in WT, ife-2 or odr-3; ife-2 animals. Left panels show GFP images and right panels show Differential Interference Contrast (DIC) images captured with a confocal microscope. The arrow points to the nuclear accumulation of DAF-16::GFP in intestinal cells of odr-3 mutant. Images were obtained using the same confocal settings and exposure adjustments were uniformly applied in all images for better visualization. 

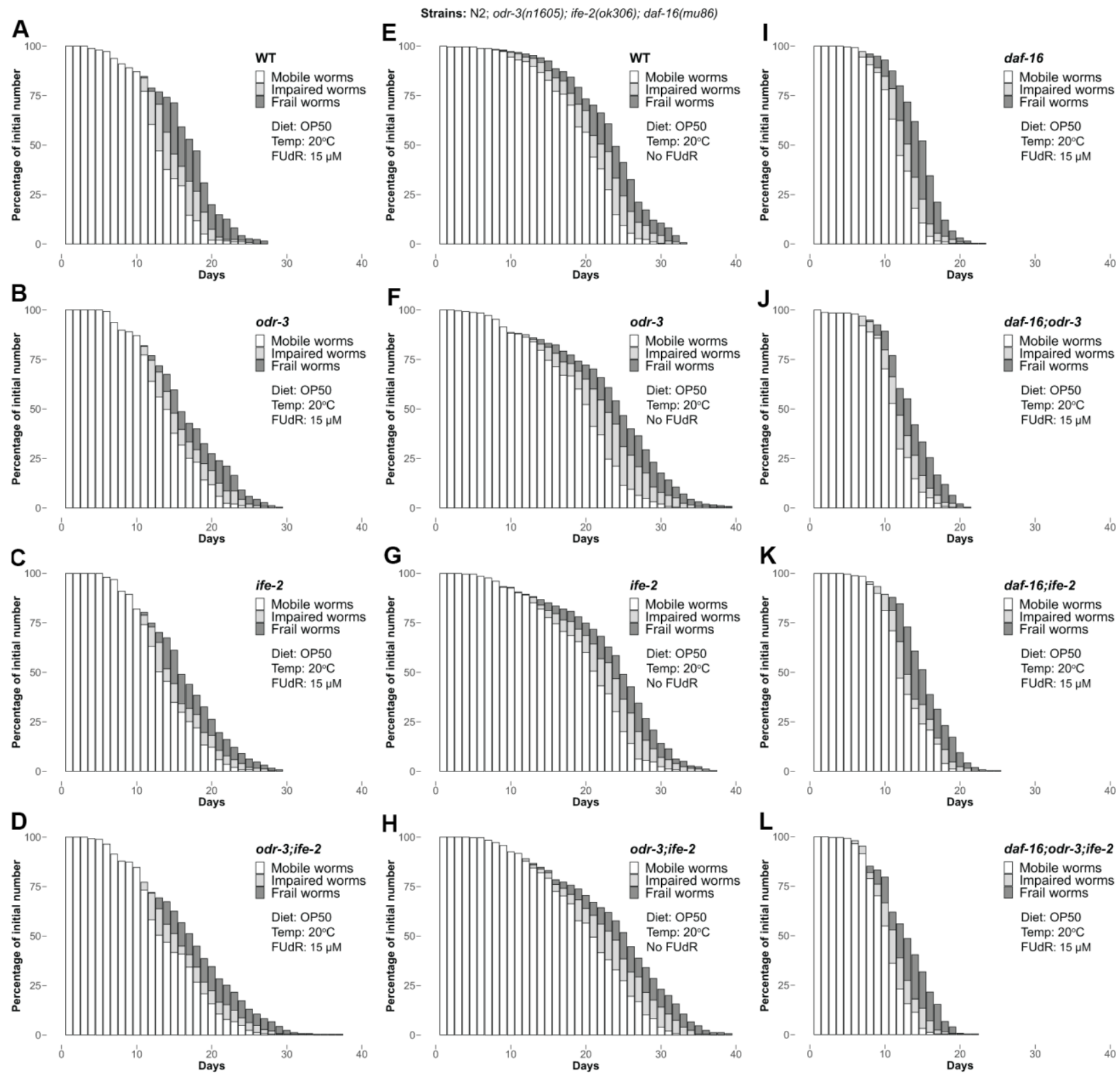

Supplementary Figure 3. Motility-assessed healthspan for mutants containing the odr-3(n1605), ife-2(ok306) and daf16(mu86) mutations. (A-L) Bar chart representation of motility-assessed healthspan illustrating the fraction of each category upon daily monitorization. Worms are grouped into three categories: mobile (white), impaired (light gray) and frail (dark gray). Dead and censored animals were subtracted from these analyses. All cohorts were kept at $20^{\circ} \mathrm{C}$ and fed OP50 E. coli. (A-D) WT, odr-3(n1605), ife-2(ok306) and odr-3(n1605);ife-2(ok306) strains on FUdR supplemented plates. (E-H) WT, odr-3(n1605), ife-2(ok306) and odr-3(n1605);ife-2(ok306) strains grown on plates with no FUdR. (I-L) odr-3(n1605), ife-2(ok306) and odr-3(n1605);ife-2(ok306) in the daf-16(mu86) background, grown on FUdR supplemented plates. 


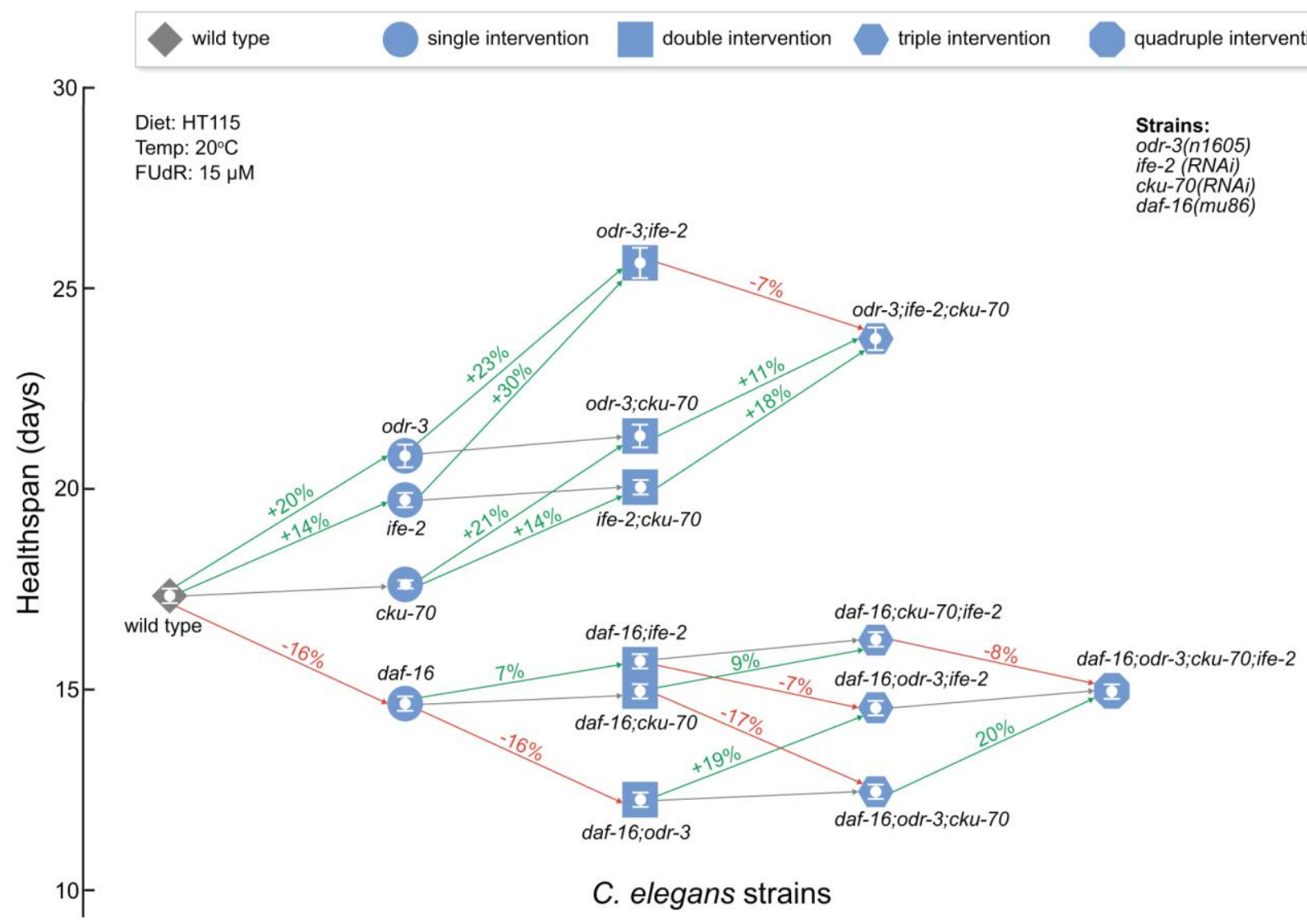

Supplementary Figure 4. Network schematic representation of the strains analyzed in this study and of the effects of each genetic intervention. Nodes represent the strains as follows: diamond for WT, circle for single gene interventions, square for double gene interventions, hexagon for triple gene interventions, and octagon for quadruple gene interventions. Nodes are positioned on the vertical axis according to their respective mean healthspan. Edges between worm strains are colored depending on the gain (or loss) in lifespan extension: increase (green), decrease (red) and small or non-significant change (gray). The extent of the change is included on the edge as a percentage increase/decrease between the origin and destination nodes of the edge. odr-3 and daf-16 denote mutants containing the odr$3(n 1605)$ and daf-16(mu86) mutations; ife-2 and $c k u-70$ denote animals in which these genes were modulated by RNAi bacteria. The white bars inside of the nodes indicate the mean \pm SEM. 

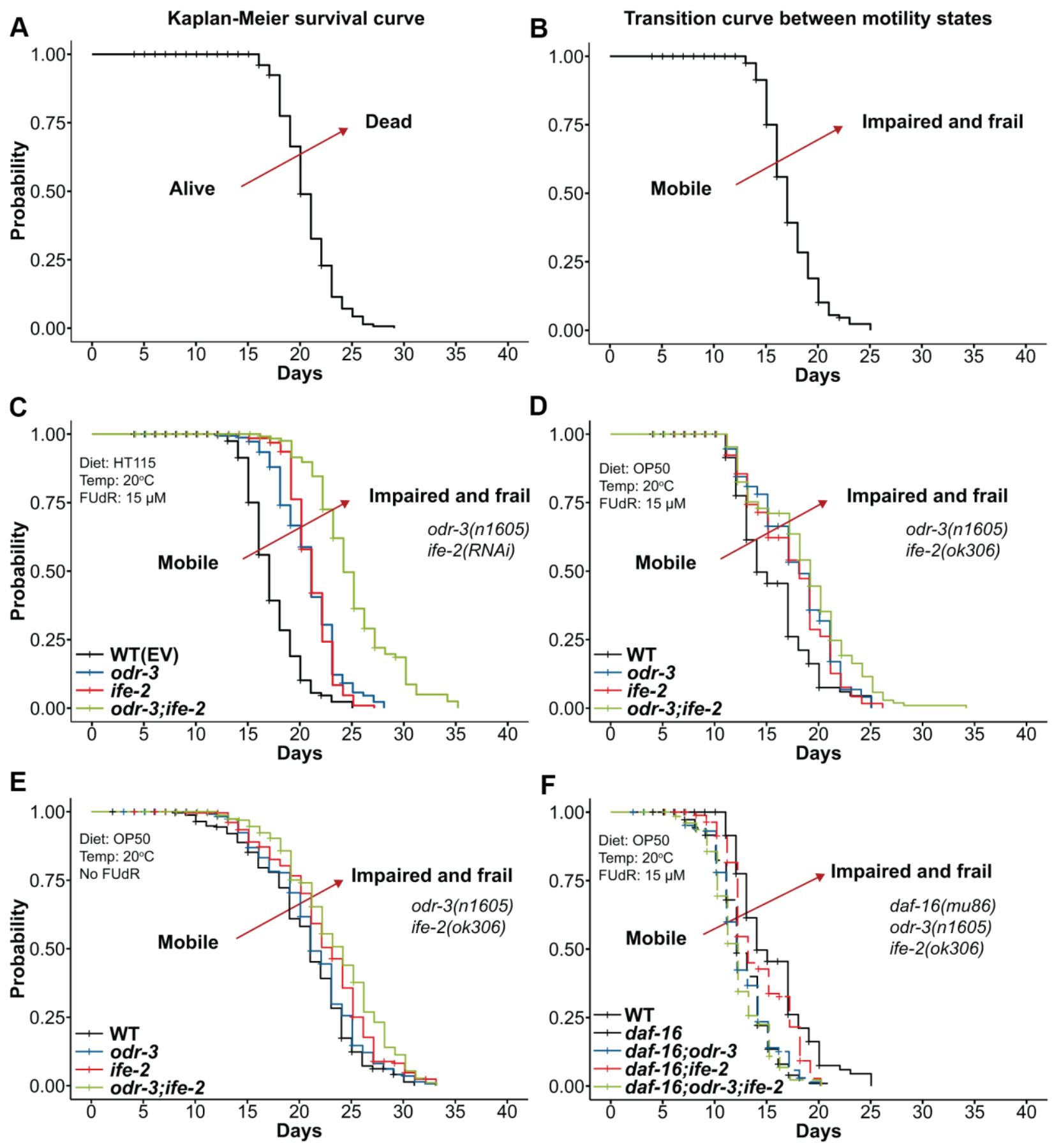

Supplementary Figure 5. Method of quantifying the statistical significance for the healthspan difference between two worm populations. Kaplan Meier curves of survival (A) and healthspan (B), showing the probability of an event occurring over time. The events represent either the death of a worm (A) or the transition from a healthy state to a sick state (becoming impaired or frail) (B). For the healthspan analysis, censored and dead worms are removed. (C-F) Comparison between healthspan curves. Statistical significance was determined using the log rank test. 


\section{Supplementary Tables}

Please browse Full Text version to see the data of Supplementary Tables 1, 2.

Supplementary Table 1. Mean, median and maximum lifespan of all worm populations assayed.

Supplementary Table 2. Mean, median and maximum number of days associated with each motility state. 